\title{
Unified Rate Theory of Electrochemistry and Electrocatalysis: Fixed Potential Formulation for General, Electron Transfer, and Proton-Coupled Electron Transfer Reactions
}

\author{
Marko M. Melander \\ Nanoscience Center, P.O. Box 35 (YN) FI-40014, Department of Chemistry, University \\ of Jyväskylä, Finland
}

\begin{abstract}
Atomistic modeling of electrocatalytic reactions is most naturally conducted within the grand canonical ensemble (GCE) which enables fixed chemical potential calculations. While GCE has been widely adopted for modeling electrochemical and electrocatalytic thermodynamics, the electrochemical reaction rate theory within GCE is lacking. Molecular and condensed phase rate theories are formulated within microcanonical and canonical ensembles, respectively, but electrocatalytic systems described within the GCE require extension of the conventionally used rate theories for computation reaction rates at fixed electrode potentials. In this work, rate theories from (micro)canonical ensemble are generalized to the GCE providing the theoretical basis for the computation reaction rates in electrochemical and electrocatalytic systems. It is shown that all canonical rate theories can be extended to the GCE. From the generalized grand canonical rate theory developed herein, fixed electrode potential rate equations are derived for i) general reactions within the GCE transition state theory (GCE-TST), ii) adiabatic curve-crossing rate theory within the empirical valence bond theory (GCEEVB), and iii) (non-)adiabatic electron and proton-coupled electron transfer reactions. The rate expressions can be readily combined with ab initio methods to study reaction kinetics reactions at complex electrochemical interfaces
\end{abstract}

Email address: marko.m.melander@jyu.fi (Marko M. Melander)

Preprint submitted to Journal of Electroanalytical Chemistry

August 9, 2019 
as a function of the electrode potential. The theoretical work herein provides a single, unified approach for electrochemical and electrocatalytic kinetics and the inclusion of non-adiabatic and tunneling effects in electrochemical environments widening the scope of reactions amenable to computational studies.

Keywords: charge transfer, Tafel slope, electrochemical kinetics, Marcus theory, grand canonical

\section{Introduction}

Electrochemical reactions and especially electrocatalysis are at the forefront of current green technologies. To realize and utilize the full potential of electrocatalysis, selective and active catalysts are needed for various applications and reactions including e.g. oxygen and hydrogen reduction/evolution reactions, nitrogen reduction to ammonia and $\mathrm{CO}_{2}$ reduction.[1] Electrochemical conversion of small molecules is most often based on successive proton-coupled electron transfer (PCET), electron transfer (ET), and proton transfer $(\mathrm{PT})$ reactions; the unique aspect of electrocatalysis is the ability to control PCET, ET, and PT kinetics and thermodynamics by the electrode potential.

Design of electrocatalysts working under complex electrochemical environments needs insight from experiments, computational methods as well as theoretical approaches.[1] Experimental techniques have reached certain maturity and tools such as potential sweep and step methods, spectroelectrochemistry, and impedance spectroscopy are standard tools for studying electrocatalytic reactions.[2] However, a similar level of maturity has not yet been reached within the computational and theoretical electrochemistry communities. Currently, there are several competing but often overlapping computational approaches available for studying reactions at electrochemical interfaces.

In experiments, the electrocatalysis is controlled by the electrolyte and electrode potential. To translate these to computationally treatable quantities, it is the combination of the electrolyte and electron electrochemical potentials which determine and control the (thermodynamic) state of electrochemical systems. Therefore, an atomic-level computational model needs to provide an explicit control and description of these chemical potentials as depicted in Figure 1. In statistical thermodynamics fixing the chemical po- 
tentials is achieved via a Legendre transformation from a canonical ensemble to a grand-canonical ensemble (GCE) for both electrons and nuclei.[3] This calls for theoretical and computational methods for treating systems in which particle numbers are allowed to fluctuate .

In electronic structure calculations as applied to electrochemical systems one of the largest difficulties is indeed modelling systems at constant electrode potentials rather than constant charges. This is a rather drastic difference and almost all electronic structure codes work exclusively for fixed charge calculations. Another difficulty faced in simulating electrochemistry is the presence of several time- and length-scales taking part in the processes. Very short time and small length-scales are needed when modelling charge transfer and chemical reactions which call for a quantum mechanical treatment of the electrode and reactants. On the other hand, the liquid electrolyte and formation of the electrochemical double-layer need a statistical treatment within GCE over a long time to properly represent the electrified solid-liquid interface. The charge distribution at the interface is controlled by the electrode potential which also directly changes both reaction kinetics and thermodynamics.

The theoretical basis for fixed potential electronic structure calculations was developed by Mermin who formulated electronic density functional theory (DFT) within GCE.[4, 5]. Later, GCE-DFT has been generalized for treating nuclear species either classically or quantum mechanically $[3,6-9]$. The GCE-DFT provides a fully DFT, atomistic approach for computing free energies of electrochemical and electrocatalytic systems at fixed electrode and ionic/nuclear chemical potentials.[3] Importantly, the free energy from a GCE-DFT calculation is in theory exact and unique to a given external potential. In practice, the (exchange-)correlation effects in both quantum and classical systems need to be approximated.

Atom-scale modeling of electrocatalytic reactions at fixed electrode $[3,10-$ $20]$ and ion potentials $[3,12,14]$ at electrochemical interfaces has been greatly advanced during the last 10-15 years and utilized in large scale studies of reactions at electrode surfaces. The work in the field of atomistic modelling of electrocatalytic reactions has been on almost exclusively focused on electrocatalytic thermodynamics. Based on the large number of theoretical and computational works utilizing GCE-DFT, the computational framework for thermodynamics within GCE seems generally accepted.

However, computation of electrochemical kinetics from atomistic simulations has remained more elusive. Like the electrochemical thermodynamics, 


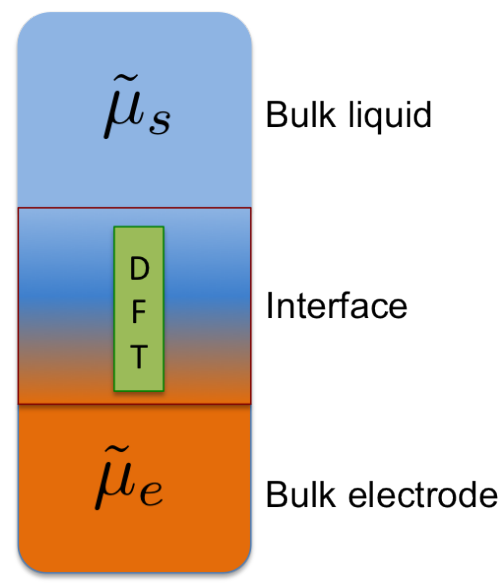

Figure 1: Pictorial model of a proper electrochemical interface at fixed electron $\tilde{\mu}_{e}$ and solvent/electrolyte $\tilde{\mu}_{S}$ chemical potentials the atomic level.

also the kinetics should be computed at fixed electrochemical potentials. This calls for generalization of fixed particle number canonical rate theories to the fixed potential GCE. Surprisingly, a general GCE rate theory has not yet been established; mending this deficiency is the central goal of the present work. As discussed in detail below, the GCE rate theory must facilitate computation of rate constants for general chemical reactions and especially PCET, ET, and PT at fixed chemical potentials. Furthermore, the theory must be applicable to both inner-sphere and adiabatic as well as outer-sphere, nonadiabatic and tunneling reactions at constant potentials. In fact, the lack of generally applicable kinetic models to treat non-adiabaticity and tunneling in electrocatalytic ET, PT, and PCET under fixed potential situations limits the scope computational and theoretical investigations of reactions to adiabatic inner-sphere reactions - a very limited subset of electrochemical and electrocatalytic reactions. This current restriction is caused by the absence of theoretical and computational methodologies[21]; while thermodynamics and kinetics of simultaneous PCET reactions are easy to evaluate for fully adiabatic inner-sphere reactions using (grand) canonical DFT and (harmonic) transition state theory (TST) vide infra, decoupled PCET reactions, outersphere ET/PT and non-adiabatic PCET reactions require more specialized approaches.

In general, ET, PT, and PCET reactions may exhibit both vibronic and electronic non-adiabaticity as well as hydrogen tunneling. The importance and contribution of non-adiabaticity and tunneling may also depend on the 
the electrode potential.[22, 23] There are several reactions where decoupled PCET i.e. separate ET and PT steps, hydrogen tunneling and nonadiabaticity have been observed. For example, in alkaline ORR pure ET has been proposed as the rate determining step[21, 24-26]. Furthermore, recent experiments of ORR on carbon-based materials show conclusively that ET is the rate- and potential-determining step. [27, 28]. On the other hand, solution $\mathrm{pH}$ can alter the reaction mechanism and ,e.g., $\mathrm{CO}_{2}$ reduction can proceed through simultaneous PCET in acidic and through decoupled PCET (ET-PT) in alkaline solutions[29, 30]. It has also been shown that only the inclusion of vibronic non-adiabaticity in electrochemical hydrogen evolution reaction can explain experimentally observed Tafel slopes and kinetic isotope effects.[22] There is also experimental evidence that room-temperature hydrogen tunneling takes place during ORR Pt and at low over-potentials tunneling is the prevalent reaction pathway.[23] Kinetics of ET are needed to describe both pure ET and decoupled PCET and in general it is expected that these pathways may prevail on weakly bonding electrode surfaces in oxygen, $\mathrm{CO}_{2}, \mathrm{CO}$, alcohol etc. reduction reactions.[31] In fact, PCET reactions are often vibronically and/or electronically non-adiabatic[32], even under electrocatalytic conditions[22].

Even though a general GCE rate theory is missing, schemes for computing rates or energy barriers of adiabatic reactions at constant electrode potentials have started to emerge. In some cases reaction barriers have been calculated explicitly at a given electrode potentials using GCE-DFT[12, 20, 3335]. However, more often various correction schemes to (Legendre) transform constant charge calculations to GCE are used for studying reaction kinetics.[11, 19, 36-39]. From both approaches the grand energy potentials as a function of the electrode potential or along the reaction coordinate are often found to exhibit quadratic dependence. This quadratic dependence of the grand energy as a function of the electrode potential has been used to transform canonical DFT barriers and reaction energies to grand energies. Recently, it has been noticed that reaction barriers as a function of the potential follow a "Marcus-like" [20] or Brønsted-Evans-Polanyi (non)linear[38] free energy relations. Other approaches for computing electrode potential-dependent barrier have relied on Butler-Volmer -type (BV) expressions where the barrier has a simple form $G(\eta)=G(\eta=0)+\alpha \eta$ where $\eta$ is the over-potential and $\alpha \in[0,1]$ is the BV symmetry factor.[38, 40, 41]. Independent of the scheme used for obtaining a constant potential reaction barrier, TST-like expressions has been used to compute rate without a sound 
theoretical basis for the validity of GCE-TST.

Even if GCE-TST proved to be valid (as it does based on the work herein), non-adiabatic and tunneling effects in ET, PT, and PCET effects would be omitted in the fully adiabatic and classical treatments. While neglecting these effects may be reasonable for many electrocatalytic reactions, all electrocatalytic reactions are certainly not inner-sphere nor adiabatic as was discussed. A handful computational and theoretical studies[22, 24, 42$48]$ at the electronic structure level have studied non-adiabaticity or tunneling effects in electrocatalytic ET/PCET. These pioneering studies utilized simplified model Hamiltonians and wave functions and computation of non-adiabatic/tunneling effects in electrocatalytic reactions. However, using general first principles methods for addressing ET/PCET kinetics have remained elusive thus far. Past theoretical and computational work on nonadiabatic electrochemical ET and PCET rates at a given electrode potentials have been accomplished using either Dogonadze-Kutzetnotsov-Levich[49, 50], Schmickler-Newns-Anderson[51, 52], or Soudackov-Hammes-Schiffer[22, 32, 45, 53-55] methods. In these treatments the electrode potential is treated as an external parameter modifying the reaction energy or barrier. These models can also incorporate electrostatic interactions between the electrode and the reactant in the double-layer. In more advanced approaches work terms and solvent reorganization energies are obtained using fixed charge molecular dynamics $[43,56]$.

When model Hamiltonian approach is combined with first principles simulations, the electronic structure, orbitals, or density of states (DOS) are computed once for a fixed number of electrons. Then, the electrode potential serves to role of changing the Fermi-level of this static electronic structure. In such calculations the electronic structure itself is considered unaltered when the potential is changed. While this might be valid in some cases, in general the electrode potential changes the solvent structure, bonding of reactants, double-layer, electronic DOS, overlap integrals etc. limiting the applicability of the static picture. Instead, modern fixed potential first-principles methods explicitly incorporate the effect of electrode potentials on the interfacial properties and bonding. Another inherent limitation occurring in previous work addressing non-adiabaticity in ET is the limitation to a single orbital picture. The traditional models assume transitions between different electrode single electron states and redox-levels of the molecule to be independent. Technically, achieving this requires separating the total wave function to filled/empty and localized orbitals. An inherent problem encountered is that 
this wave function separation cannot be achieved without additional assumptions as shown in Section 1 of the Supporting Information. In practice this hampers the computation of ET rates from DFT or wave function methods because an additional (and rather) arbitrary orbital separation/localization step is required. A general electrocatalytic rate theory should not be restricted to model (single-orbital) wave functions or Hamiltonians. Instead, a many-electron wave function obtained using $a b$ initio methods at a fixed potential should be used to capture the inherent complexity reactions at electrochemical interfaces. In the canonical ensemble, ET, PT, and PCET rates of electronically and vibronically (non-)adiabatic reactions can be studied using either model or general Hamiltonians[32, 54, 55, 57-61]. Extending these canonical rate theories to fixed potential GCE is the direction pursued herein. This is important from both practical and conceptual point of views that electronic and vibronic non-adiabaticity and tunneling can be included in electrochemical, fixed potential ET, PT and PCET rates using generalized Hamiltonians, many-electron wave functions, and rate theory.

The above discussion highlights that electrochemical (outer-sphere) and electrocatalytic (inner-sphere) reactions have treated using different approaches. Commonly, electrocatalytic reactions have been studied using adiabatic TST theory while electrochemical reactions have relied on perturbative non-adiabatic theories. However, in the canonical ensemble, all rate theories equally applicable to inner- or outer-sphere reactions can be derived using a single general framework provided by Miller[62-64]. To enable an equally well-defined and generally valid rate theory in an electrochemical setting, in this work I have extended Miller's (micro)canonical rate theories to electrochemical systems at fixed chemical potentials described within the GCE. The formulation presented herein is equally applicable to electrocatalytic and electrochemical reactions and, hence, presents a general unified approach. This includes the possibility to account for tunneling as well as vibronic and electronic non-adiabaticity, for example. While methods for treating thermodynamics, locating transition states and energy barriers within GCE have been devised, a general method for computation reaction rates - not just barriers - has not yet available. The GCE rate theory enables the use of all canonical rate theories in constant potential simulations.

In this work, the general framework is developed and utilized to derive rate constants for adiabatic ET, PT and PCET reactions using a generalized GCE Marcus-like [65] empirical valence bond theory (GCE-EVB). The non-adiabatic ET and PCET rate constants are derived using a golden-rule 
formalism within GCE. The theoretical work results in ET and PCET rate constants valid for both adiabatic and non-adiabatic (proton-coupled) electron transfer rates and the inclusion of proton tunneling in PCET. The developed rate theories can readily be combined with modern computational methods based on (GCE-)DFT. The fixed potential rate theory will expand the type of systems, conditions, and phenomena in electrocatalysis amenable for first principles modelling.

The paper is organized as follows. In Section 2 a general rate theory and TST within GCE are developed. Rest of the paper focuses on ET and PCET kinetics using GCE-TST. Section 3 shows how the adiabatic barrier and rate of ET and PCET reactions are computed using GCE-EVB and free energy perturbation theory within GCE leading to a fixed potential version of Marcus theory. Tafel slopes and other use quantities as extracted from GCE-EVB are analyzed. In section 4 non-adiabatic rate constants for ET and PCET reactions with generalized first-principles Hamiltonians and manyelectron wave functions. In section 5 computational aspects for evaluating the rate constants are discussed.

\section{Rate theory in the grand canonical ensemble}

As highlighted in the preceding discussion, the electrode potential is expected to affect the energetics and kinetics in complex ways. Thus, the potential should be treated explicitly rather than as a simple corrective parameter as often done in theoretical and computational models used in electrocatalysis. Formulating all expectation values within GCE naturally includes the electrode potential from the start and this forms the basis for the methods developed here and building on our previous grand canonical multi-component DFT[3]. The key is that the electrode potential is included in the $a b$ initio Hamiltonian within the GCE and as results all observables and quantities depend explicitly on the potential. For details on GCE, see Section 2 of the Supporting Information and previous work in Ref.3.

To extend (micro)canonical rate theory to the GCE, only particle conserving reactions are considered. Thus, only a state with $N$ particles can be converted to state with $N$ particles but the population and probability of $N$ particle states is determined by the GCE density operator. Hence, all equilibrium quantities are always well-defined but jumps between states with unequal number of particles are suppressed. In general this is not expected to limit the applicability of the rate expressions derived in this work; 
if a quantum system is characterized by particle conserving operators $(\hat{H}$ Hamiltonian, $\hat{S}$ entropy, and $\hat{N}$ particle number), even time-dependent observables are obtained as ensemble weighted expectation values from $O(t)=$ $\operatorname{Tr}\left[\hat{\rho} \hat{U}\left(t_{0}, t\right) \hat{O}(t) \hat{U}\left(t, t_{0}\right)\right]=\sum_{n} p_{n}\left\langle\psi_{n}\left|\hat{U}\left(t_{0}, t\right) \hat{O}(t) \hat{U}\left(t, t_{0}\right)\right| \psi_{n}\right\rangle$ which do not include changes between states with different number of particles.[66] Hence, even explicit propagation of the wave function does not allow sudden jumps in particle numbers or jumps between states between different number of particles.

In a similar way, particle fluxes needed for the flux formulation of rate theory (see below) can be applied within the GCE as long as (local) equilibrium is maintained. This implies that the Hamiltonian is time-independent and that only particle conserving reactions contribute to the rate constant according to the grand canonical distribution[67]. Furthermore, computation of correlation functions and hence fluxes poses both theoretical and computational difficulties. While both may in principle be directly computed within GCE[67], the computation includes the coupling of the system to the particle reservoir and introduces the reservoir time scales. Also, the sampling should only include trajectories for which the particle number is equal at times $t$ and $t+\tau$. This is because in GCE the phase space volume is not globally conserved and Liouville theorem does not hold. As a result, the computed ensemble properties will depend on time if the system is not in equilibrium i.e. the phase space distribution function $\rho(\mathbf{q}, \mathbf{p}, N, t)$ is not stationary[67-69] ( $d_{t} \rho(\mathbf{q}, \mathbf{p}, N, t) \neq 0$ and $\mathbf{p}$ and $\mathbf{q}$ are momentum and position, respectively). In the context of the present work it is important to notice that both equilibrium $\left(d_{t} \rho(\mathbf{q}, \mathbf{p}, N, t)=0\right.$ at $\left.t \rightarrow \infty\right)$ and instantaneous $\left(\lim _{t \rightarrow 0^{+}}\right)$properties are uniquely defined by the GCE[67, 69]; both qualities are absolutely essential in order to formulate the rate and transition state theories within GCE.

Herein only equilibrium and instantaneous quantities are used. Intermediate times would require running GCE-dynamics or making assumptions on the reservoir-system couplings. Hence, non-equilibrium processes cannot be treated using the approaches presented in this paper. Another limitation of the current approach is that kinetics of electron transfer from the electron "bath" degrees of freedom are not included and are therefore assumed sufficiently fast. Neither of these limitations are should greatly limit the applicability of the approach for electrocatalytic or electrochemical reactions. In these reactions the electron bath is provided by a conducting electrode and 
equilibrium conditions are controlled by constant temperature and potential which also provide the natural control parameters in the GCE utilized in this work. It is noted that mass transfer in electrochemical systems is not in equilibrium or even steady-state. However, the reaction rate coefficients are independent of particle fluxes and concentrations and therefore the elementary rate constants can be characterized by their equilibrium values as long as the Hamiltonian of the quantum part is time-independent and particle conserving.

After establishing the particle conserving and equilibrium nature of the rate constants, the GCE rate constants can be formulated. To allow various types of reactions to be described, the canonical rate expression due to Miller [62-64, 70] is adopted:

$$
k(T, V, N) Q_{I}=\int d E P(E) \exp [-\beta E]=\lim _{t \rightarrow \infty} C_{f s}(t)
$$

where $Q_{I}$ is the canonical partition function of the initial state, and $\beta=\left(k_{B} T\right)^{-1}$. The first expression is written in terms of transition probability at a given energy $P(E)$. Second expression utilizes the canonical flux-side correlation function $C_{f s}(t)=\frac{1}{(2 \pi \hbar)^{f}} \int d \mathbf{p}^{f} d \mathbf{q}^{f} \exp (-\beta H) \delta[f(\mathbf{q})] \dot{\mathbf{q}} h\left[f\left(\mathbf{q}_{t}\right)\right.$ for $f$ degrees of freedom. $\delta[f(\mathbf{q})]$ constrains the trajectories to start from the dividing surface, $\dot{\mathbf{q}}$ is the initial flux along the reaction coordinate, and $h\left[f\left(\mathbf{q}_{t}\right)\right]$ is the side function which includes the dynamic information whether a trajectory is reactive or not. Based on the discussion above, only the $t \rightarrow 0^{+}$ and $t \rightarrow \infty$ should be considered for the flux-side correlation function in the rate expressions. The rate from either the transition probability and fluxside formulations are equivalent. Depending on the choice of $P(E)$ or $H$ and $h[f]$ non-adiabatic and adiabatic (nuclear) quantum effects are included in the rate. $[71,71-74]$

To compute reaction rates at fixed potentials a straight-forward, yet novel, extension of the canonical rate theory to the GCE is made:

$$
k(T, V, \mu) \Xi_{I}=\sum_{N=0}^{\infty} \exp [\beta \mu N] Q_{0}(T, V, N) k(T, V, N)
$$

where $\Xi_{I}=\exp [\beta \mu N] Q_{I}$ is the initial state grand partition function and $k(T, V, N)$ was introduced in Eq. (1). Above $N$ is the number of species (nuclear or electronic) in the system. 
While the above equations are completely general and various flavors of rate theories can be extracted by invoking different Hamiltonians and transition probabilities, they are somewhat cumbersome to treat. Indeed, it would be convenient if the GCE could be used directly to evaluate the rate without explicitly sum over different particle numbers. This can be achieved by introducing the transition state theory (TST) assumption[62-64] but generalized to GCE herein. In canonical TST, the instantaneous $\lim _{t \rightarrow 0_{+}} C_{f s}(t)$ is considered corresponding to the assumption that there are no-recrossings of the dividing surface. Both quantum/classical and adiabatic/non-adiabatic TSTs are written as [75-78]

$$
k_{T S T}(T, V, N) Q_{0}(T, V, N)=\lim _{t \rightarrow 0_{+}} C_{f s}(t)
$$

and the exact rate is recovered by introducing a correction

$$
\begin{array}{r}
k(T, V, N)=\lim _{t \rightarrow \infty} \kappa(t) k_{T S T}(T, V, N) \\
\text { with } \quad \kappa(t)=\frac{C_{f s}(t)}{C_{f s}\left(t \rightarrow 0_{+}\right)}
\end{array}
$$

where $\kappa(t)$ is the time-dependent transmission coefficient. For long-times it can also be written as $\kappa=k(T, V, N) / k_{T S T}(T, V, N)$.[79] Inserting this equation in Eq.(2) can be used to compute the most general grand canonical rate constant. To further simplify the treatment, below I will focus on classical nuclei unless explicitly stated. As shown in the SI section 3, for classical nuclei the TST results is $[63,64]$ :

$$
\begin{aligned}
& k(T, V, \mu) \Xi_{I}=\sum_{N=0}^{\infty} \exp [\beta \mu N] \int d E P_{c l}(E) \exp [-\beta E] \\
& \approx \sum_{N} \exp [\beta \mu N] \frac{k_{B} T}{h} Q^{\dagger} \equiv \frac{k_{B} T}{h} \Xi^{\dagger}
\end{aligned}
$$

\footnotetext{
Above, $P_{c l}(E)$ denotes transition probability for classical nuclei but the electrons are of course quantum mechanical $[60,80]$ and the details can be found in Ref. 64 and the SI. The result of the previous equation shows that the structure of GCE-TST and canonical TST are similar. This is true for open system in general if memory effects are neglected[81]. To obtain the
} 
GCE rate constant without invoking the TST approximation one can use the transmission coefficient to write

$$
k(T, V, \mu) \Xi_{I}=\sum_{N=0}^{\infty} \exp [\beta \mu N] \kappa(T, V, N) \frac{k_{B} T}{h} Q^{\ddagger} \approx\left\langle\kappa_{\mu}\right\rangle \frac{k_{B} T}{h} \Xi^{\ddagger}
$$

where it is assumed that the transmission coefficient is insensitive to changes in the particle number and $\left\langle\kappa_{\mu}\right\rangle$ is the effective transition probability. To complete the derivation for the classical GCE rate constant, the rate is expressed in terms of grand energies

$$
k(T, V, \mu)=\left\langle\kappa_{\mu}\right\rangle \frac{k_{B} T}{h} \exp \left[-\beta \Delta \Omega^{\ddagger}\right]
$$

where the definition $\Omega_{i}=-\ln \left(\Xi_{i}\right) / \beta$ has been used and $\Delta \Omega^{\ddagger}=\Omega^{\ddagger}-\Omega_{I}$ is the GCE barrier. Above the only new assumption besides grand canonical equilibrium distribution and the TST, is that the flux out of the transition state does not depend on the number of particles in the system, i.e. the $\kappa$ can be treated as a constant. For large enough systems and small variations in the particle this a well justified assumption.

The above development establishes a general fixed chemical potential rate theory. Within the TST approximation the rate is determined by the grand free energy barrier. The transmission coefficient needs to be approximated but this depends on the case at hand. The adiabatic and non-adiabatic harmonic GCE-TSTs expression for the fully open system are derived in Supporting Information section 3.

\subsection{Explicit dependence only on electron chemical potential}

The development above is valid when both nuclear and electronic subsystems are open. A significant simplification results if one assumes that the reaction rate is does not explicitly depend on the number of some nuclei in the system. In a typical first principles calculation this simplification can be exploited if one assumes that the system can be divided to two subsystem: 1) classical electrolyte species consisting of nuclei and electrons and 2) electrode + reactants treated either classically or quantum mechanically. Typically the number of nuclei constituting the electrode and reactant are fixed while the electrolyte chemical potential needs to be fixed. The electrolyte charge density also adjusted to maintain charge neutrality of the system. 
Fixing only the electron and electrolyte chemical potentials gives a semigrand canonical ensemble used for deriving the thermodynamics of electrocatalytic systems in Ref. 3. Within the semi-grand canonical ensemble, the electrode+reactants set the external potential at a fixed electrode potential while the electrolyte adapts to changes the thermodynamics and to maintain charge neutrality; the electrolyte is at a fixed chemical potential but the energetics to do not explicitly depend on the number of electrolyte species. In this case, summation over the number of electrode/reactant nuclei or the electrolyte species is not needed. This is also the typical scheme used in first principles modelling within GCE and Poisson-Boltzmann models, for example.

Herein the semi-GCE is applied to derive rate equations as a function of electrode potential. From now on, I assume that reaction rates depend explicitly only on the number and/or chemical potential of electrons in the system. Then, the state of the system is determined by $T, V$, number of nuclei of the electrode+reactant $N_{N}$, chemical potential of the electrolyte, chemical potential of the electrons $\mu_{n}$, and number of electrons in the system $N$ unless explicitly specified otherwise. Electroneutrality is maintained by the electrolyte. Harmonic TST rates for constant number of nuclei and constant electrochemical potentials are derived in section 3 of the Supporting Information.

\section{Adiabatic barriers and rates from GC-EVB}

To compute the GCE-TST rate at a given electrode potential, the grand energy barrier of Eq. (7) needs to be obtained. For electronically adiabatic reactions methods like the constant-potential[20] nudged elastic band[82] can be used. However, usually one is interested in rates as a function of the electrode potential and, hence, the barrier needs to be obtained for a range of electrode potentials which is computationally expensive.

As shown below, an alternative method for computing the grand energy barrier is to formulate a Marcus-like[65] approach within GCE. Marcus theory can be viewed as special case of the empirical valence bond (EVB) theory [83] commonly utilized in electron [65] and proton transfer theories. [53, 83-86] Using a novel extension of thermodynamic perturbation theory to the GCE setting, a GCE-EVB has been derived in this work (see SI sections 4 and 5). The GCE-EVB theory developed herein provides a theoretically well-justified and computationally affordable way for computing fixed poten- 
tial barriers at various electrode potentials; the adiabatic barrier needs to be explicitly computed only at a single electrode potential while barriers at other potentials can be obtained using well-defined interpolation of Eq.(16).

In these EVB and Marcus theories the initial and final states are presented using diabatic states, effective wave functions and free energies[65]. This can be extended to GCE by using two effective, fixed potential surfaces which can be understood as a statistical mixture of states with probabilities given by the density operator in GCE (see Section of 2 the Supporting Information and our previous work in 3). Importantly, the diabatic ground states obtained using the GCE density operator naturally include many-body effects of the coupled electrode-reactant-solvent system and the complexity of the electrochemical interface is explicitly included in the model. Also, there is no need to decompose the rate constants to orbital dependent quantities; in the current GCE formulation, the redox-molecule and the electrode are fully coupled and the total wave function $|r, \mathbf{e}\rangle$ is treated as a single entity in (see Section 1 in the Supporting Information for additional discussion). Then, two grand canonical diabatic all-electron wave functions are used to form an effective diabatic GCE Hamiltonian. This is analogous to molecular Marcus theory in which the canonical diabatic Hamiltonian comprises of an initial (oxidized) $I$ and final(reduced) molecule $F$.

Following the treatment in the Supporting Information Section 4, an effective $2 \times 2$ grand canonical Hamiltonian in Eq. (8) can be formed. The resulting form is analogous to the canonical empirical valence bond[83] (EVB) used in electron [65], proton[85, 86] and proton-coupled electron[53] theories. The present form is, however, crucially different from its predecessors; based on the approach developed in this work, in all quantities are defined and computed at fixed electrode potential using the GCE.

$$
H_{G C E-d i a}=\left[\begin{array}{cc}
\Omega_{I I} & \Omega_{I F} \\
\Omega_{F I} & \Omega_{F F}
\end{array}\right]
$$

where the diagonal elements are the grand energies of the oxidized (II) and reduced (FF) systems. The off-diagonal elements account for the interaction and mixing of the initial and final states. In this, way the off-diagonal elements can be fitted so that diagonalization of Eq.(8) produces the adiabatic grand canonical potential energy surface.

Finally, note that the (diabatic) grand canonical states correspond to a single electron density which is guaranteed by the Hohenberg-Kohn-Mermin [3, 
4] to be unique for a given electrode potential. If a general quantum mechanical Hamiltonian is used, bond breaking is naturally included in the GCE-EVB model. The only disambiguity is the definition of these diabatic states. In principle it is also possible to add other, possibly excited states as basis states. In practice the GCE diabatic energies, $\left(\Omega_{I I}\right.$ and $\left.\Omega_{F F}\right)$, can be computed directly by applying using e.g. constrained DFT[87-89] with fixed potential DFT as discussed in Section 5. Below it is shown how the grand canonical free energies can be obtained from atomistic simulations.

\subsection{Computation of diabatic GCE surfaces and barriers}

An approach often used in molecular simulations for constructing the diabatic free energy curves is to sample the diabatic potentials along a suitable reaction coordinate. For ET, PT, and PCET reactions in the canonical ensemble this coordinate is the energy gap between the two diabatic states as shown by Zusman[90] and Warshel[91]: $\Delta E_{\text {gap }}(R)=E_{F}(R)-$ $E_{I}(R) .[68,92]$ From the sampled energy gap the free energy curves are obtained as $A(R)=-k_{B} T \ln \left(p\left(E_{\text {gap }}(R)\right)\right)+c$. If the distribution is Gaus$\operatorname{sian}\left(p\left(E_{\text {gap }}(R)\right)=c \exp \left[-\left(\Delta E_{\text {gap }}-\left\langle\Delta E_{\text {gap }}\right\rangle\right)^{2} / 2 \sigma^{2}\right]\right)$ and the resulting free energy curves a parabolic. The barrier in EVB or Marcus theory is then obtained from the intersection of the initial and final diabatic curves[92-95].

Within GCE, the energy gap is simply $E_{g a p}(R ; \mu)=\sum_{N, i} p_{N, i} E_{g a p}\left(R_{i}, N\right)$. As shown in the SI section 5, the gap distributions can be formulated and computed by generalizing Zwanzig's[96] canonical free energy perturbation theory to the GCE. This route provides a rigorous way to derive the reaction barrier in terms of diabatic states and energies as presented in the Supporting Information Section 5. The reaction energy barrier can be computed from the initial-final state energy gap distribution functions using[91, 97-102]

$$
k_{I F}=\kappa \frac{\exp \left[-\beta g_{I}\left(\Delta E^{\ddagger}\right)\right]}{\int d \Delta E \exp \left[-\beta g_{I}(\Delta E)\right]}=\kappa p_{I}\left(\Delta E^{\ddagger}\right)
$$

where $g_{i}(\Delta E)$ is the free energy curve in state $i$ as a function of the energy gap, $p_{I}\left(\Delta E^{\ddagger}\right)$ is the gap distribution at the transitions state, and $\kappa$ denotes an effective pre-factor. The above shows that the reaction rate is determined by the energy gap distribution function $p^{I}(\Delta E)=\langle\delta(\Delta E(R)-\Delta E)\rangle_{I}$ from Eq. (30) of the Supporting information.

When assuming that $E_{\text {gap }}(R ; \mu)$ is Gaussian, the GC-diabatic states are parabolic and the Marcus barrier in GCE is given by Eq. (12). As shown 
in the Sections 5 of the SI, the (Gaussian) gap distribution may be derived using a (second order) cumulant expansion. This results in gap distribution of the following form

$$
p_{I}(\Delta E)=\frac{1}{\sqrt{2 \pi \sigma_{I}}} \exp \left[-\frac{\left(\Delta E-\langle\Delta E\rangle_{I}\right)^{2}}{2 \sigma_{I}^{2}}\right]
$$

where $\langle\Delta E\rangle_{I}$ is the energy gap expectation value in the initial state obtained from Eq.(S31) in the Supporting Information and $\sigma_{I}=\left\langle(\Delta E)^{2}\right\rangle_{I}-$ $\left(\langle\Delta E\rangle_{I}\right)^{2}$ is the gap variance. The Marcus relation is then obtain after standard manipulations $[92,98]$ yielding

$$
p_{I}\left(\Delta E^{\ddagger}\right)=\frac{1}{\sqrt{4 k_{B} T \Lambda}} \exp \left[-\beta \frac{\left(\Delta \Omega_{F I}+\Lambda\right)^{2}}{4 \Lambda}\right]
$$

where $\sigma_{I}^{2}=\sigma_{F}^{2}=2 k_{B} T \Lambda=k_{B} T\left(\langle\Delta E\rangle_{I}-\langle\Delta E\rangle_{F}\right), \Lambda$ is the reorganization grand energy and and $\Delta \Omega_{F I}=\frac{1}{2}\left(\langle\Delta E\rangle_{I}+\langle\Delta E\rangle_{F}\right)$ is the reaction grand energy as depicted in Figure3.1. Finally, the Marcus expression within GCE is

$$
k=\frac{\kappa}{\sqrt{4 k_{B} T \Lambda}} \exp \left[-\beta \frac{\left(\Delta \Omega_{F I}+\Lambda\right)^{2}}{4 \Lambda}\right]
$$

The energy barrier of Eq. (12) is the diabatic energy barrier. The adiabatic barrier can the be computed using Eq. (8) as discussed in Section. 3.2 below. One caveat to keep in mind is more involved computation of $\kappa$ within the GCE as shown in Section 4. The above result may safely be used when $\kappa \approx 1$ for all particle numbers meaning that the reaction is always fully adiabatic.

\subsection{Implications of the canonical GCE-EVB rate theory}

For symmetric grand energy surfaces the diabatic grand energy barrier can be is estimated from the crossing point of the two quadratic grand energy surfaces with equal curvatures is given in Eq. (12). Adopting the work Mattiat and Richardson[103] on the canonical ensemble, the assumption on equal curvature can be relaxed by specifying an asymmetry parameter $\alpha_{a s}$ as

$$
\alpha_{a s}=\frac{\Lambda_{I}-\Lambda_{F}}{\Lambda_{I}+\Lambda_{F}}
$$




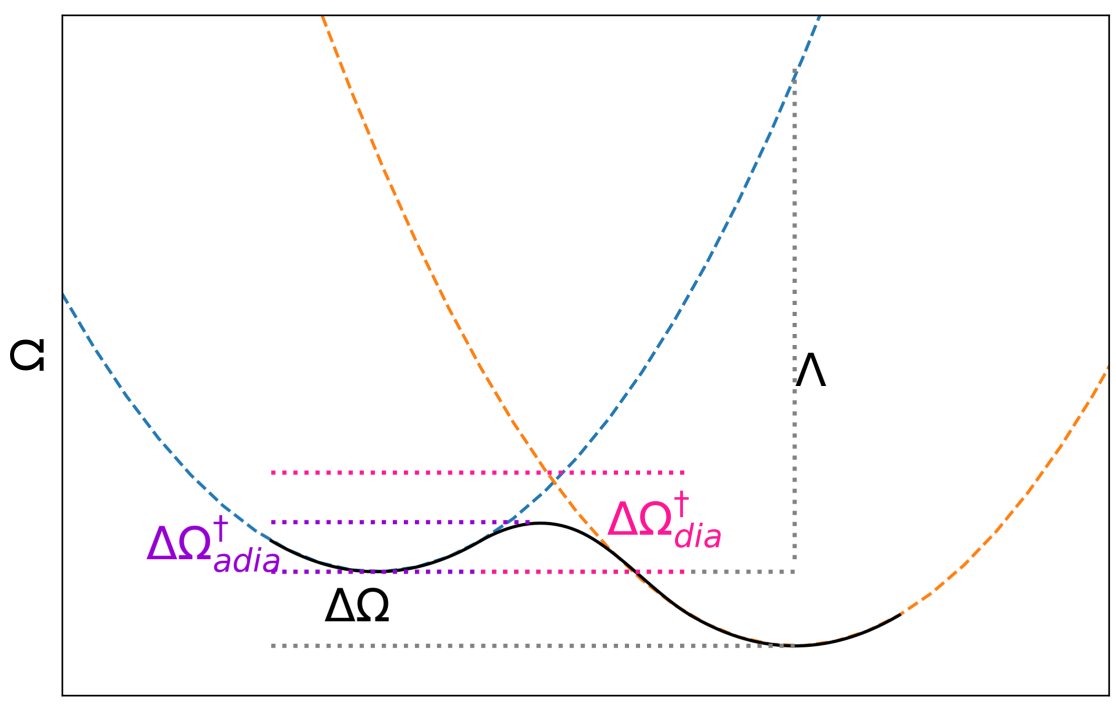

Reaction coordinate $(x / \xi)$

Figure 2: Schematic depiction of the important GCE-EVB quantities. The blue (orange) dashed lines is initial (final) diabatic surface while the black solid line is the adiabatic surface.

in terms of the reorganization energies for both the initial and final states

$\Lambda_{I}$ and $\Lambda_{F}$, respectively. The transition state is located at the crossing point

$$
x^{\ddagger} / \xi=-\frac{1}{\alpha_{a s}}+\frac{1}{\alpha_{a s}} \sqrt{1-\alpha_{a s}\left(\alpha_{a s}+\frac{4 \Delta \Omega}{\Lambda_{I}+\Lambda_{F}}\right)}
$$

Using these definitions the asymmetric diabatic Marcus barrier and rate are obtained as

$$
\begin{gathered}
\Delta \Omega^{\ddagger}=\frac{1}{4} \Lambda_{I}\left(x^{\ddagger} / \xi-1\right)^{2} \\
k \approx \frac{\kappa}{\sqrt{4 k_{B} T \Lambda_{I}}} \frac{1+\alpha_{a s}}{1+\alpha_{a s} x^{\ddagger} / \xi} \exp \left[-\beta \Delta \Omega^{\ddagger}\right]
\end{gathered}
$$

If $\alpha_{a s} \rightarrow 0$, the regular Marcus rate and barrier are obtained. In Fig.3.2 the effect of asymmetry and reaction energy to the reaction barrier and location of the transition state are compared. It can be seen that both the 
barrier heights and its location are affected by the asymmetry and reaction energy.
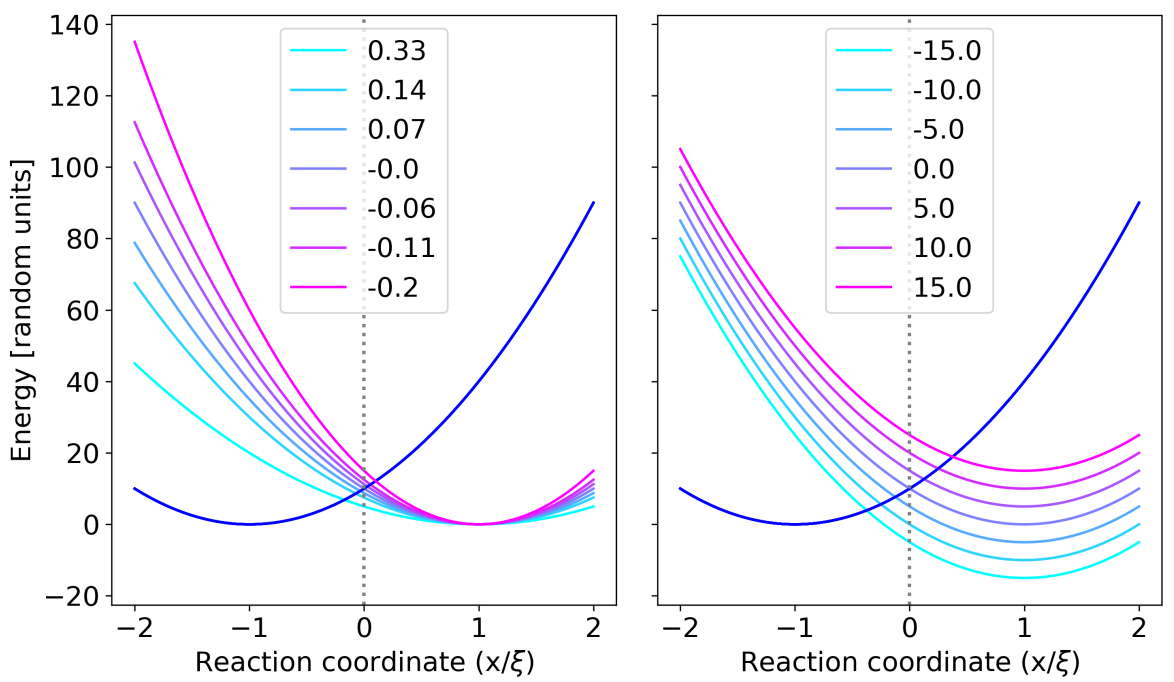

Figure 3: Left: EVB curves at different different asymmetries $\alpha_{a s}$. The initial state reorganization energy is $\Lambda_{I}=40$ while the final state reorganization energy $\Lambda_{F} \in[20,60]$. The reaction energy is $\Delta \Omega=0$ for all curves. Right: EVB curves as a function of the reaction energy: $\Delta \Omega \in[-15,15]$. For all curves $\Lambda_{I}=\Lambda_{F}$. Both: The dashed line at $x=0$ indicates the position of the transition state when $\Lambda_{I}=\Lambda_{F}$ and $\Delta \Omega=0$. The curve crossing point equals $\Delta \Omega_{d i a}^{\ddagger}$

While the Marcus-like equation results in a diabatic barrier, the adiabatic reaction barrier can be extracted from the diabatic barrier obtained by diagonalizing Eq.(8) or from .(12) by introducing an adiabaticity correction. For the canonical ensemble, this correction is known as the Hwang-ÅqvistWarshel equation[104]. If the GCE-diabatic states are quadratic along the reaction coordinate and share the same curvature along the reaction coordinate, the adiabatic barrier can be written as $[104,105]$

$$
\begin{aligned}
\Delta \Omega_{a d, E V B}^{\ddagger} & =\frac{(\Delta \Omega+\Lambda)^{2}}{4 \Lambda}-\Omega_{I F}\left(x^{\ddagger}\right)+\frac{\left(\Omega_{I F}\left(x^{I}\right)\right)^{2}}{\Delta \Omega+\Lambda} \\
& =\Delta \Omega_{d i a}^{\ddagger}-\Omega_{I F}\left(x^{\ddagger}\right)+\frac{\left(\Omega_{I F}\left(x^{I}\right)\right)^{2}}{\Delta \Omega+\Lambda}
\end{aligned}
$$

where $\Omega_{I F}$ is the off-diagonal matrix of the GCE-EVB Hamiltonian in Eq. (8). If the Condon approximation is used, the above equation is greatly 
simplified as $\Omega_{I F} \approx \Omega_{I F}\left(x^{\ddagger}\right) \approx \Omega_{I F}\left(x^{I}\right)$. From a practical perspective it is interesting to observe how the adiabatic GCE-EVB barrier changes when the parameters a changed. From the schematics shown in Figures 3.1 and 3.2, one can observe that changes of the minima along the reaction coordinate correspond to horizontal displacements of the diabatic states and and changes in $\Lambda$. Vertical changes correspond to changes in the reaction grand energy $\Delta \Omega$. Usually one concentrates only on changes in the free energy as reorganization coordinate not expected change for similar reactions or different electrode potentials (this assumptions is also made in Section 4.) Focusing on the reaction grand energy, it is easy show that under equilibrium conditions, $\Delta \Omega=0$, the barrier is given by

$$
\Delta \Omega_{a d, E V B}^{0, \ddagger}=\frac{\Lambda}{4}-\Omega_{I F}+\frac{\left(\Omega_{I F}\right)^{2}}{\Lambda} \approx \frac{\Lambda}{4}-\Omega_{I F}
$$

which leads to $\Lambda=4\left(\Delta \Omega_{a d, E V B}^{0, \ddagger}+\Omega_{I F}\right) \approx 4 \Delta \Omega_{\text {dia }}^{0, \ddagger}$ assuming that $\Omega_{I F}<<$ $\Lambda$. The equilibrium point is characterized by zero over-potential $\eta=\Delta \Omega=0$. Replacing the solution for $\Lambda$ in Eq. (16) gives the diabatic barrier as

$$
\Delta \Omega_{\text {dia }}^{\ddagger}=\Omega_{\text {dia }}^{0, \ddagger}\left(1+\frac{\Delta \Omega}{4 \Omega_{\text {dia }}^{0, \ddagger}}\right)^{2}=\Delta \Omega_{\text {dia }}^{0, \ddagger}+\frac{\Delta \Omega}{2}+\frac{(\Delta \Omega)^{2}}{16 \Delta \Omega_{\text {dia }}^{0, \ddagger}}
$$

Inserting (18) in (16) results in the adiabatic reaction barrier as

$$
\Delta \Omega_{a d, E V B}^{\ddagger}=\Delta \Omega_{a d, E V B}^{0, \ddagger}+\frac{\Delta \Omega}{2}+\frac{(\Delta \Omega)^{2}}{16 \Delta \Omega_{d i a}^{0, \ddagger}}
$$

This result has several interesting implications and connections to previous work. The most immediate is that at small changes in the driving force $\Delta \Omega$, a linear dependence between the barrier and reaction energy is established. However, at larger driving forces, a non-linear dependence appears.

This can be directly translated to the language of electrochemistry by considering the changes in driving force as a function of the electrode potential or over-potential. As discussed by Trasatti[106, 107] and in our recent work[3], the absolute electrochemical potential and chemical potential are related by $E^{M}(a b s) \sim-\tilde{\mu}_{n}$ independent of the reference scheme. It is important to notice that for microscopic systems usually considered within GCE-DFT keeping $\tilde{\mu}_{n}$ fixed leads to changes in the number of electrons in the initial and final states. As a result the canonical free energies $A(N)$ do 
not remain constant when change when $\tilde{\mu}_{n}$ is changed. Therefore, changes in the grand energy is in general $\delta \Omega=A\left(N_{F} ; \tilde{\mu}\right)-A\left(N_{I} ; \tilde{\mu}\right)-\tilde{\mu}_{n}\left(N_{I}-N_{F}\right)$.

$\delta \Omega$ may be extracted from constant potential calculations enabling the study of electrochemical kinetics as a function of the electrode potential: $-\partial r\left(T, V, \tilde{\mu}_{n}\right) / \partial \tilde{\mu}_{n}$ as done in a Tafel analysis, for example. The traditional measure in electrochemistry for reaction kinetics is the Tafel slope measuring how current is affected by changes in the over-potential. In heterogeneous and homogeneous catalysis the corresponding quantity is the Brønsted-EvansPolanyi (BEP) coefficient or more generally (linear free) energy relations measuring the change of reaction rate when the reaction energy is changed. However, the work of Fletcher[108, 109] and Parsons[110] show that Tafel and BEP type analyses actually measure the same quantities; both measure the reaction rate as a function of the changes in the reaction driving force. For macroscopic electrochemical reactions the driving force is measured in terms of the over-potential while in microscopic calculations the driving force is the free energy. These two quantities are linked by $|\Delta \eta|=\left|\Delta \tilde{\mu}_{n}\right|=|\Delta \partial \Omega / \partial n|$.

Both the BEP and Tafel coefficients maybe computed from a single expression. The Tafel coefficient is defined as[2, 108, 109]

$$
\alpha \propto \frac{\partial \ln k}{\partial E}=-\frac{\partial \ln k}{\partial \Delta \Omega} \frac{\partial \Delta \Omega}{\partial \tilde{\mu}_{n}} \frac{\partial \tilde{\mu}_{n}}{\partial E}=-\gamma \Delta \Omega^{\prime}
$$

where $\gamma$ is BEP relationship and $\Delta \Omega^{\prime}$ denotes the grand energy change as a function of the over-potential. Also $E \sim \tilde{\mu}_{n}$ has been used.

Let us focus first on the $\Delta \Omega^{\prime}$ term which depends on the reaction and needs to be approximated. To facilitate this analysis, one recognizes that $\Delta \Omega=\left(A_{F}\left(\left\langle N_{F}\right\rangle\right)-A_{I}\left(\left\langle N_{I}\right\rangle\right)-\tilde{\mu}_{n}\left(\left\langle N_{F}\right\rangle-\left\langle N_{I}\right\rangle\right)\right.$. For macroscopic systems, i) chemical reactions have $N_{F}=N_{I}$ while ii) simple electrochemical steps have $N_{F}=N_{I} \pm 1$. Then for chemical reactions $\Delta \Omega=\Delta A$ and the variation $\Delta \Omega^{\prime}$ is expected to be small. For electrochemical reactions at the macroscopic limit, a particularly straightforward estimate is obtained from the computational hydrogen electrode (CHE) concept.[111] In the CHE model, the reaction energy $\Delta \Omega \approx \Delta A^{0} \mp \eta$ for PCET steps with $\Delta A^{0}$ computed without any bias potential. Hence, within CHE, $\alpha=\gamma$ for PCET steps and zero otherwise. Similar reasoning holds also for simple (outer-sphere) ET reactions in macroscopic systems as shown in Section 6 of the SI. For these reactions $\Delta \Omega \approx \Delta A^{0} \mp$ constant $\times \eta$ and $\Delta \Omega^{\prime}=\mp$ constant.

For microscopic systems, however, such a simple relationship does not hold in general and models such as GCE-DFT can be used for computing 
$\Delta \Omega^{\prime}$ explicitly. Thus far, $\Delta \Omega^{\prime}$ has been reported in only few studies $[20$, 112]. In both works, $\Delta \Omega$ exhibits a roughly linear dependence on the applied potential. To conclude, $\Delta \Omega^{\prime}$ is expected to be a constant close to unity for electrochemical reactions and close to zero for chemical reactions.

Next, the BEP $\gamma$ of Eq (20) is analyzed. Using the diabatic barriers, one obtains

$$
\gamma=\left.\frac{\partial \ln k\left(T, V, \tilde{\mu}_{n}\right)}{\partial \Delta \Omega}\right|_{T, V}=\left[\frac{1}{2}+\frac{\Delta \Omega}{8 \Delta \Omega_{\text {dia }}^{0, \ddagger}}\right]=\frac{1}{2}\left[1+\frac{\Delta \Omega}{\Lambda}\right]
$$

From the above equation, it is seen that $\gamma$ is not a simple constant but depends linearly on the reaction driving force. If the reorganization energy is small the dependence on the reaction grand energy becomes more pronounced. Based on the generalized BEP-Tafel energy identities the following relationships can be observed:

- If the quadratic part in Eq.(18) is neglected, one obtains the ButlerVolmer (BV) barrier. In this case the barrier depends linearly on the applied potential as $\Delta \Delta \Omega_{d i a, E V B}^{\ddagger} \approx 0.5\left(A_{F}\left(\left\langle N_{F}\right\rangle\right)-A_{I}\left(\left\langle N_{I}\right\rangle\right)-\right.$ $\mu_{e l}\left(\left\langle N_{F}\right\rangle-\left\langle N_{I}\right\rangle\right) . \mu_{e l}$ is implicitly referenced against $\mu_{e l}^{e q}=0$ and can easily be converted to the over-potential $\mu_{e l}-\mu_{e l}^{e q}=\Delta \eta$. Note that $\Delta \Delta \Omega_{d i a, E V B}^{\ddagger}$ is not expected to be linear for finite-sized systems.

Again, for macroscopic systems $\left\langle N_{F}\right\rangle=\left\langle N_{I}\right\rangle$ and $\Delta \Delta \Omega_{d i a, E V B}^{\ddagger}=\Delta \Delta A_{\text {dia,EVB }}^{\ddagger}=$ $0.5\left(A_{F}-A_{I}\right)$ which is the Brønsted-Evans-Polanyi result. The BV relationship is obtained by treating a specific reaction type. For example, in a typical ET, PT, or PCET the potential-dependent reaction free energy is given by $\Delta A=\Delta A(\eta=0) \pm(n \eta)$. Using this for $\Delta \Delta A= \pm 0.5 n \eta$.

- Non-linearity of the grand energy barrier was already established above. For macroscopic systems non-linearity is established by including the quadratic part of the diabatic barrier in model. Lately[20, 36, 38] this has been observed computationally and it is pleasing that the GCEEVB picture seems qualitatively correct.

A spectacular feature of canonical Marcus and EVB theory is the observation of an inverted region i.e. the rate constant starts to decline as the reaction becomes more exothermic. However, the inverted region has not been observed for electrochemical reactions even at large over-potentials. The grand 
canonical Marcus rate of Eq. (12) seems to predict an inverted region for highly exothermic conditions. However, as written in the Tafel equation (20) the rate as a function of the over-potential depends on both the change in A) barrier as a function of the reaction energy and B) change reaction energy as a function of the over-potential. A) would indeed predict an inverted region but B) suppress this if $\Delta \Omega \approx 0$. Then the Tafel slope would approach zero as predicted by the Marcus-Hush-Chidsey[113], Dogonadze-Levich-Kuztnetsov[49, 50], Newns-Anderson-Schmickler, Soudackov-Hammes-Schiffer[45] models of ET and PCET [51] (see also Supporting Information Section 1). At the moment, there is not enough computational nor theoretical evidence on the behaviour of $\Delta \Omega$ as a function of the over-potential to predict or to analyze the Tafel slope any further. Also for very small barriers, reorganizational dynamics of the surroundings may start to limit the reaction and dynamical properties of the surroundings need to addressed as discussed in Section 5.

To summarize, the generalized BEP-Tafel relationships has been derived from a microscopic perspective starting from a grand canonical rate theory. Both variation in the reaction energy barrier and the transition state location as a function of the potential can be predicted using just a few parameters. The general form of the BEP-Tafel relation is given in Eq. (20). For small over-potentials, the rate is expected to depend linearly on the applied potential. For larger over-potentials non-linear dependence is predicted, especially reactions for which the reorganization energy is small.

\section{Non-adiabatic ET and PCET reaction rates within GCE}

As shown above, computation of electronically adiabatic reaction rates from either GCE-HTST, GCE-EVB or GCE-perturbation theory do not yield any fundamental difficulties as compared to the canonical case; after finding the barrier, one can simply use a simple TST-like expression to compute the reaction rate using grand free energies. However, for a non-adiabatic process, using the grand free energy is not as straightforward.

The main difficulty becomes from computation of the electronic transition matrix element which is not defined for states with different number of electrons. Hence, one cannot directly use the effective GCE-EVB states developed in Section 3 and use them to compute the electronically nonadiabatic rate. Instead, in rigorous setting, the electronic transition matrix element needs to be computed separately for each canonical transition. Afterwards, a summation over the canonical rates is performed to express 
the non-adiabatic ET/PCET rate as a expectation value. To obtain the non-adiabatic TST rate, the Golden-rule approach is used herein. In the canonical ensemble, the Golden-rule formulation of the rate is equivalent to Dogonadze's treatment. $[49,50,93]$ Below theory for the computation of non-adiabatic ET and PCET rates within GCE is developed.

\subsection{Non-adiabatic ET rate}

To start with, the electronic states $|i N\rangle$ are specified and they are eigenstates to the electronic Hamiltonian $\hat{H}_{N}^{e l}$. Electronic states are defined for initial $(i)$ and final $(f)$ states with a fixed number of particles $(N)$. Then the electronic energies for the initial and final states at fixed particle number at nuclear geometry $Q$ are

$$
\left\langle i N\left|\hat{H}_{N}^{e l}\right| i N\right\rangle=\varepsilon_{i N}(Q) \quad \text { and } \quad\left\langle f N\left|\hat{H}_{N}^{e l}\right| f N\right\rangle=\varepsilon_{f N}(Q)
$$

Within the Born-Oppenheimer approximation (BOA), the nuclear wave functions and their energies $\epsilon$ in the initial $(|m N\rangle)$ and final $(|n N\rangle)$ electronic states are obtained from

$$
\begin{aligned}
& {\left[\hat{T}_{Q}+\varepsilon_{i N}(Q)\right]|m N\rangle=\epsilon_{m N}|m N\rangle \text { and }} \\
& {\left[\hat{T}_{Q}+\varepsilon_{f N}(Q)\right]|n N\rangle=\epsilon_{n N}|n N\rangle}
\end{aligned}
$$

where $\hat{T}_{Q}$ is the nuclear kinetic energy. Within BOA, the total vibronic wave function and the corresponding energy factorize as

$$
\begin{aligned}
|i m N\rangle & =|i N\rangle|m N\rangle \quad \text { and } \quad E_{i m N}=\varepsilon_{i N}+\epsilon_{m N} \\
|f n N\rangle & =|f N\rangle|n N\rangle \quad \text { and } \quad E_{f n N}=\varepsilon_{f N}+\epsilon_{n N}
\end{aligned}
$$

As the different energy contributions are additive, the canonical partition functions can be factorized:

$$
\begin{aligned}
& Q_{i}^{N}=\exp \left[-\beta \varepsilon_{i N}\right] \sum_{m} \exp \left[-\beta \epsilon_{m N}\right] \quad \text { and } \\
& Q_{f}^{N}=\exp \left[-\beta \varepsilon_{f N}\right] \sum_{n} \exp \left[-\beta \epsilon_{n N}\right]
\end{aligned}
$$




$$
\begin{aligned}
k_{G C E-N A T S T} & \approx \frac{2 \pi}{\hbar \Xi_{i}} \sum_{N} e^{-\beta\left(\varepsilon_{i N}-\mu N\right)} \sum_{m, n} \frac{e^{-\beta \epsilon_{m N}}}{Q_{m}}\left|\left\langle N n f\left|\hat{V}_{N}\right| i m N\right\rangle\right|^{2} \delta\left(E_{i m N}-E_{f n N}\right) \\
& =\frac{2 \pi}{\hbar} \sum_{N} p_{i N} \sum_{m, n} p_{m N}|\langle N n f|\hat{V}| i m N\rangle|^{2} \delta\left(E_{i m N}-E_{f n N}\right)
\end{aligned}
$$


where $p_{i N, e l}=\exp \left[-\beta\left(\varepsilon_{i N}-\mu N\right)\right] / \Xi_{i, e l}$ and $p_{m N}=\exp \left[-\beta \epsilon_{m N}\right] / Q_{m}$.

This equation has the structure of the canonical Golden rule rate weighted by the probability of being in the initial electronic state $i N$. To simplify the notation, one can momentarily concentrate only on the canonical part of the above rate expression. As shown in the Supporting Information Section 7, using the Fourier transform presentation of the delta function, gives

$$
k_{G C E-N A T S T} \approx \sum_{N} \frac{V_{N, f i}^{2}}{2 \hbar^{2}} p_{i N} \int d t C(t)
$$

where $C(t)$ is an energy autocorrelation function (see Supporting Information Section 7). The autocorrelation function maybe extracted from timedepenendent quantum or classical dynamics. However, to obtain a closed form for the rate equation, herein the autocorrelation function is expressed using a cumulant expansion[114]. Using the second order cumulant expansion, assuming that all solvent degrees of freedom are classical and taking the short time approximation [115] to the correlation function results in (see Supporting Information Section 7):

$$
k_{G C E-N A T S T} \approx \sum_{N} p_{i N} \frac{V_{N, i f}^{2}}{\hbar \sqrt{4 \pi k_{B} T \lambda}} \exp \left[-\frac{\left(\Delta E_{f i}^{N}+\lambda\right)^{2}}{4 k_{B} T \lambda}\right]
$$

The reorganization and reaction energies are defined as $\lambda=E_{i m}\left(Q_{F}\right)-$ $E_{f n}\left(Q_{F}\right)$ and $E_{f i}^{N}=E_{f n}^{N}\left(Q_{F}\right)-E_{i m}^{N}\left(Q_{I}\right)$ (see Figure 3.2.) The reorganization energy can be further separated to inner and outer sphere components as discussed in Section 10 of the Supporting Information. If this separation is invoked, one can alleviate the assumption that the total reorganization is independent of the particle number and instead assume that only bulk solvent (outer sphere) reorganization is a constant while the inner-sphere reorganization energy depends on the particle number.

\subsection{PCET kinetics within $G C E$}

The PCET kinetics is based on the PCET rate theory of Soudackov and Hammes-Schiffer. Within the canonical ensemble the relevant rate expressions were derived in Refs. 45, 53-55 and here this treatment is extended to the GCE yielding PCET rate constants at fixed electrode potentials. The PCET rate constant derivation follows a similar procedure as the one used 
above for the ET rates. In the case of PCET, an additional geometric variable $q$ to denote the position of the transferring proton is introduced. Within $\mathrm{BOA}$, the total vibronic wave function is then

$$
|i u m N\rangle=|i N(q, Q)\rangle|u N(Q)\rangle|m N\rangle
$$

where it is explicitly written that the electronic wave function $|i N\rangle$ depends explicitly on the proton $q$ and system coordinate $Q$ while the proton wave function $|u N(Q)\rangle$ depends on the system coordinate $Q$. The wave functions and corresponding energies are solved using equations similar to the ET case

$$
\begin{gathered}
\left\langle i N\left|\hat{H}_{N}^{e l}\right| i N\right\rangle=\varepsilon_{i N}(q, Q) \quad \text { and } \\
\left\langle f N\left|\hat{H}_{N}^{e l}\right| f N\right\rangle=\varepsilon_{f N}(q, Q) \\
{\left[\hat{T}_{q}+\varepsilon_{i N}(q, Q)\right]|i u N\rangle=\epsilon_{u N}^{i}|i u N\rangle \quad \text { and }} \\
{\left[\hat{T}_{q}+\varepsilon_{f N}(q, Q)\right]|f v N\rangle=\epsilon_{v N}^{i}|f v N\rangle} \\
{\left[\hat{T}_{Q}+\epsilon_{u N}^{i}\right]|m N\rangle=\mathcal{E}_{m N}|m N\rangle \quad \text { and }} \\
{\left[\hat{T}_{Q}+\epsilon_{v N}^{f}\right]|n N\rangle=\mathcal{E}_{n N}|n N\rangle}
\end{gathered}
$$

where $\hat{T}_{q}$ and $\hat{T}_{Q}$ are the kinetic energy operators for the proton and other nuclei, respectively. Within BOA, the total energy of the at fixed $N$ is written as a simple sum of the three contributions:

$$
E_{\text {iumN }}=\varepsilon_{i N}+\epsilon_{u N}^{i}+\mathcal{E}_{m N}
$$

and similarly for the final diabatic state. Furthermore, coupling constant is given as

$$
\left\langle N n v f\left|\hat{V}(R)_{N}\right| i u m N\right\rangle \approx\left\langle N v f\left|\hat{V}(R)_{N}\right| i u N\right\rangle_{q}\langle N n \mid m N\rangle_{Q}=V(R)_{u v}^{N} S_{n m}^{N}
$$

The SHS treatment of PCET rates is valid for reactions ranging from vibronically non-adiabatic to vibronically adiabatic scenarios[116] and rate expressions for various well-defined limits have been achieved. The SHS PCET rate theories are derived following a path analogous to the derivation of ET rates and extension to the GCE is rather straightforward. As done by SHS, the Golden rule formulation is used. The details of this derivation 
are presented in the SI Section 11. The simplest GCE-PCET rate is given for the short time approximation of the energy gap correlation is valid in the high-temperature limit and static proton donor-acceptor $R$ distance as

$$
k=\sum_{N, u} p_{i u} \sum_{v} \frac{\left|V(R)_{u v}^{N}\right|^{2}}{\hbar \sqrt{4 \pi k_{\mathrm{B}} T \lambda_{u v}}} \exp \left[-\frac{\left(\Delta E_{u v}^{N}+\lambda_{u v}\right)^{2}}{4 k_{\mathrm{B}} T \lambda_{u v}}\right]
$$

where the reaction energy between vibrational states $i u N$ and $f v N$ is $E_{u v}^{N}=E_{f v n N}\left(q_{F}, Q_{F}\right)-E_{\text {ium }}\left(q_{I}, Q_{I}\right)$. The state-dependent reorganization energy $\lambda_{u v}=E_{\text {ium }}\left(q_{F}, Q_{F}\right)-E_{f v n}\left(q_{F}, Q_{F}\right)$ is assumed independent of the particle number. If some vibrational modes (besides the $R$ mode) are sensitive to changes in the particle number, they can be separated from the total reorganization energy by decomposing the total reorganization energy to inner- and outer-sphere components as shown in Section 10 of the Supporting Information. Depending on the form of the prefactor, both electronically and vibronically adiabatic and non-adiabatic limits of PCET can be reached within the semiclassical treatment $[22,117,118]$ of the prefactor.

\subsection{Analysis of the non-adiabatic GCE rates}

The main difficulty observed in the GCE non-adiabatic rate theory is the treatment of the electronic/vibronic coupling constant; this term is defined only for particle conserving transitions. This precludes the straightforward use of GCE diabatic states which have different number of electrons at the same geometry. Only at the thermodynamic limit when the particle number fluctuation is zero can the GCE diabatic states be used for computing the coupling constant. However, at this limit the GCE-NATST is equal to the canonical NATST as only a single particle number state is populated i.e. $p_{i}$ becomes a delta function around some particle number. At thermodynamic limit either using fixed potential GCE states or fixed particle number canonical states will give equivalent results, as they should.

Even at the thermodynamic limit the present treatment differs from the traditional Dogonadze-Kutzetnotsov-Levich[50], Schmikler-Newns-Anderson[51, 52], and SHS approaches. A detailed discussion is given in Section 1 of the Supporting Information and here only the main differences are high-lighted. The crucial difference is that the present formulation does not rely on the separation of the total interacting wave function to non-interacting or weakly interacting fragments. Also, in the present approach, the applied electrode 
potential does not only affect the electrode alone but rather modifies the entire systems affecting all electrode, reagent, and solvent species. Hence, the inherent complexity of the electrochemical interface is naturally included in the Hamiltonian and the wave function from the start. For instance, the work terms entering Marcus[65] or other electrochemical rate theories[56, 119] do not need to be computed when using the present formalism. Another crucial difference is that the charge transfer kinetics are not decomposed into single electron orbital contributions. Instead, the work herein formulates the kinetics in terms of many-body diabatic wave functions. In the canonical ensemble, such an approach has been shown[120] to provide accurate barriers, prefactors, and overall kinetics for electron transfer reaction in battery materials.

For small systems where particle number fluctuations are pronounced the summation over particle numbers need to be performed. While straightforward in principle, the amount of calculations can seem daunting at first. However, as the populations depend exponentially on the energy and target chemical potential, $p_{i N} \sim \exp \left[-\beta\left(E_{i N}-\mu N\right)\right]$, only a limited number of states will contribute to the summation. In Section 8 of the Supporting Information, it is shown that for graphene, the electrode potential around the $\mathrm{PZC} \pm 0.5 \mathrm{~V}$ is accurately captured using seven different charge states. It is expected that the infinite summation can be safely reduced to summation over a small number (5-10) of different charge states covering the electrode potential range of interest. Again, at the thermodynamic limit only a single calculation per potential is needed.

\section{Discussion}

The fixed potential rate theory developed herein does not utilize model Hamiltonians. Instead, all the above rate equations can be parametrized and evaluated directly using first principles atomic simulations with general Hamiltonians. As there is no need to parametrize the model Hamiltonians, adoption and evaluation of the rates is straight-forward (but potentially laborious).

There are a few special requirements for the software used for parametrizing the rate equations. First, simulation of charged systems is needed to sample the electrode potential. Electroneutrality can be enforced using some variant of the Poisson-Boltzmann models, for details see Ref. 3. Fixed potential calculations can be accomplished within a single SCF cycle[10], or 
iteratively [20, 121]. Second, the solvent effects should be included in the model. While adiabatic reactions can in principle be modeled without solvent contributions, the solvent is known to greatly affect the stability of reaction intermediates and should therefore be included for qualitatively and quantitatively accurate calculations. Computation of non-adiabatic reaction rates should always be performed in the presence of a solvent; the reaction barrier is directly related to the solvent/environment reorganization energy and neglecting the solvent contributions will most likely lead to incorrect results.

Given a software capable of handling charged systems and performing constant potential calculations, adiabatic rate constants can be readily evaluated. One only needs to compute the adiabatic constant potential reaction barrier using e.g. the NEB[82] method. Evaluating non-adiabatic and GCEEVB rate constants requires additional software capabilities for constructing charge/spin localized diabatic states and to evaluate the electronic coupling between these states. Also the reorganization energy, which is an excited state quantity, needs to be computed. One widely implemented and available tool for evaluating the additional parameters is the constrained DFT methodology[87-89] which is implemented in several DFT codes[122-132]. Evaluation of the vibronic/vibrational matrix elements is accomplished using e.g. a Fourier grid Hamiltonian[133] method which is easy to implement. GCE-EVB simulations should be accompanied with a constant potential simulation to compute fixed potential reaction and reorganization energies. Nonadiabatic rate constants need sampling at different charge states to evaluate the summation over the number of electrons. While this summation is in principle infinite, in practice only 5-10 charge states suffice because GCE weight is non-zero only for a few states as discussed in Section 4.3.

The presented formalism is highly appealing as it enables treating of electrochemical and electrocatalytic kinetics and thermodynamics[3] within a single formalism - the GCE. Therefore, the same code and set of DFTbased tools can be used to address inner-sphere and outer-sphere kinetics and thermodynamics instead of modifying or changing the theoretical and computational framework for different reaction steps is done in e.g Ref. 24. Also, the derived rate equations can be self-consistently parametrized using e.g. DFT calculations directly at the electrochemical interface. For instance, evaluation of the coupling matrix elements does not rely on orbitalto-orbital transitions and integration over the DOS as done in traditional non-adiabatic perturbation theory -based approaches[52] (see Supporting In- 
formation Section 1). Also the evaluation of chemisorption functions used for computing interaction strengths and energies in the adiabatic NewnsAnderson-based models[52, 119] is avoided in the current approach. Therefore, the current models are free of approximate treatment of the DOS using semi-elliptic bands[119, 134] or fitting the chemisorption functions to a computed DOS[134].

As the presented approach does not rely on any specific Hamiltonian, the computed energies can capture the interplay between the electronic structure, solvent, electrode potential etc. The electrode potential is self-consistently treated and all free energies depend explicitly on the potential. This is in contrast with traditional treatments where the electrode potential rigidly shifts the Fermi-level without modifying any interactions[52, 56] or modifying only electrostatic interactions[22, 119]. Also, a separate computation of work terms $[56,119]$ is not needed because all relevant interaction can be directly included in the general Hamiltonian. Furthermore, unlike Newns-Anderson[135] or perturbation theories[136], the current rate theory nor a general Hamiltonian need to be modified to account for bond making/breaking events as these are implicitly described through the general quantum mechanical Hamiltonian. Studying adiabatic reactions involving ET, PT, or PCET and bond rupture/formation using GCE-DFT is straightforward. Bond formation in non-adiabatic reactions is also captured by diabatic models using DFT as demonstrated for ET[120], PCET[137] and general chemical reactions[138, 139].

A final computational aspect in applying the current approach is the interpolation between the different rate equations. Such an interpolation is needed to bridge the adiabatic and non-adiabatic rate constants because the division between inner-sphere/electrocatalytic/adiabatic and outer-sphere/nonadiabatic/electrochemical is not always straightforward. Also such a division depends on the reaction, reaction conditions as well as e.g. distance between the reactant and the electrode. Such an interpolation is also needed for describing the kinetics of activationless reactions in which the rate and degree of solvent/surrounding reorganization energy determine the reaction rate[140]. Currently, a generally valid interpolation for fixed potentials has not yet been developed. In the canonical ensemble interpolation between electronically/vibrationally adiabatic and non-adiabatic reactions can be accomplished using the Landau-Zener formula.[58, 92]. In PCET, a universally valid interpolation from a fully non-adiabatic to fully adiabatic reaction is accomplished using a semiclassical PCET prefactor[117]. 
Besides interpolating between the adiabatic and non-adiabatic limits, the interpolation to reactions where solvent dynamics set the time-scale relevant should be considered. The solvent dynamics are likely to be increasingly important when the reaction becomes adiabatic and the reaction barrier becomes very small or vanishes. In these limits the solvent reorganization will be the slowest process and the reaction prefactor should reflect this. Within the canonical ensemble and in the electronically non-adiabatic limit interpolation to solvent controlled reactions is usually based on the works of Zusman[90] or Rips and Jortner[140]. In the electronically adiabatic limit the solvent dynamics are often described in terms of the Kramers-Grote-Hynes theory[141]. While numerous attempts have been taken[142-144] to obtain a universally valid interpolation between adiabatic - solvent dynamic -nonadiabatic, the author is not aware a generally accepted construction for this interpolation. Also adapting the interpolation schemes to the fixed-potential rate theory needs requires care. More work is obviously needed to obtain a robust interpolation between well-defined limits in an electrochemical setting.

\section{Conclusions}

In this work a new theoretical formulation is developed for computation electrochemical and electrocatalytic rate constants at a fixed potential. Also computational aspects for evaluating the newly developed rate equations are thoroughly discussed. Ways to address e.g. adiabatic, non-adiabatic, and tunnelling reactions can be formulated within GCE and are discussed through-out the work. Specifically, the grand canonical rate formulation is applied to derive rate constants for i) general electrocatalytic reactions with (Eq. (7)) and without (Eq. (2)) the TST approximation, ii) electronically adiabatic ET, PT and PCET reactions using a grand canonical Marcus-like GCE-EVB theory in Eq. (12), and iii) non-adiabatic ET in Eq. (31) and PCET in Eq. (36). Future work will provide interpolation between the derived adiabatic, non-adiabatic, and solvent-controlled rate equations.

The fixed-potential rate constants are based on a novel formulation obtained by extending the universally valid canonical rate theory[62-64] to the grand canonical, fixed potential ensemble. Section 2 derives the general conditions and limitations for the fixed potential rate theory. It is then shown that all rate theories developed within the canonical ensemble can be extended to GCE. This is conceptually important because the fixed-potential rate theory enables treating all potential-driven reactions within a single 
formalism instead of relying on separate theories for electrocatalysis (ButlerVolmer or adiabatic Marcus theories) and electrochemistry (Dogonadze-Kutzentsov, Schmikler, Gerischer, non-adiabatic Marcus theories). The theoretical work presented herein provides a unified framework for computing and understanding both inner-sphere and outer-sphere reaction kinetics as a function of the electrode potential.

In addition to the conceptual appeal, the present approach has also several practical advantages. First, the theoretical framework enables the use of general Hamiltonians to compute the reaction rates at fixed potentials. Notably, the developed theory can be directly combined with modern, solidstate $a b$ initio methods to capture the complexity of the electrochemical interface. In this sense, the model is fully $a b$ initio and all parameters can be directly computed without resorting to fitting. A set of widely implemented DFT-based tools suffices to compute all the needed parameters in a selfconsistent manner. This enables the computational community to progress from a thermodynamics-based description of electrocatalysis to addressing also electrocatalytic kinetics in experimentally realistic conditions.

In its most general form, the fixed potential rate theory requires computation of canonical rates for a set of systems with a varying number of electrons (and/or nuclei). Summing and weighting the different canonical ensemble rates can be relaxed if one assumes that the prefactor or transmission coefficient is independent on the number of particles in the system. Assuming a constant transmission coefficient directly leads to TST like equations (Eqs. (6) and (7)) where the reaction rate depends exponentially on the grand energy barrier $\Delta \Omega^{\ddagger}$. This is most useful and provides the theoretical basis for computing adiabatic reaction rates within GCE-TST as done in several recent publications $[12,19,20,35,36,145,146]$ in which the rate expression was used without a priori justifying the use of such rate equations.

Further insight in the (electronically adiabatic) reaction rates and energy barriers is obtained from a Marcus-like, grand canonical ensemble empirical valence bond (GCE-EVB) theory developed in the present work. As shown in Section 3, the GCE-EVB formulation enables writing the grand energy barrier in terms of fixed potential reorganization energy and the reaction grand energy in analogy with the canonical EVB or Marcus theory. As discussed in Section 3.2, this formulation enables computation and rationalization of both non-linear grand energy relationships and Tafel slopes. Together these may called BEP-Tafel relations. Both can be derived, analyzed and computed using just a few parameters which can be obtained using e.g. a combination of 
fixed potential and constrained DFTs. Based on the BEP-Tafel relationships one determine how the reaction barrier changes as a function of the reaction energy as shown in Figure 3.2. The derived adiabatic GCE-EVB rate, barrier and generalized BEP-Tafel energy relation predict and explain the "Marcuslike" behavior in energy barriers as a function of the thermodynamic driving force observed in recent computational work[20, 36, 38].

To go beyond TST, fixed potential rate constants are derived also for electronically (and vibronically) non-adiabatic ET and PCET reactions. Thus far, computational work on non-adiabatic effects and pure ET have remained scarce due to methodological difficulties despite their practical importance in providing new reaction pathways to avoid constraining scaling relations[147149] encountered for adiabatic PCET reactions while predicting catalytic activity as well as in understanding fundamental phenomena in electrocatalysis. The NA-ET rate constants derived herein will especially useful for studying NA effects in outer-sphere ET and PCET in electrocatalytic systems. This provides means to obtain atomic-level insight on pure ET reactions which have remained elusive and neglected in computational studies but have often been observed experimentally, especially on weakly-binding catalysts, as discussed in Section 1. The fixed potential PCET rate equations facilitate the study of kinetics of ubiquitous proton-coupled electron transer reactions. As formulated herein, the PCET rate constant naturally includes both electronic and vibronic non-adiabaticity as well as hydrogen tunneling. This again enables detailed theoretical and computational studies of these experimentally observed, but thus far computationally largely neglected, electrocatalytic reactions.

Combining the presented rate theory with currently existing GCE-DFT methods and various solvation models is straight-forward and enables the study of electrochemical and electrocatalytic kinetics at realistic electrochemical interfaces. This will greatly improve our microscopic understanding by enabling computation of electrocatalytic kinetics as a function of the electrode potential and addressing tunneling and non-adiabaticity in electrocatalysis. Hence, a wide variety of mechanistic, kinetic and thermodynamic aspects of electrocatalytic reactions can be addressed on equal footing within GCE and the complex interplay between the electrode potential, solvation, double-layer and electrocatalysis can be studied from first principles. Besides providing a rigorous and general theoretical framework for fixed potential kinetics, the advances herein enable computational studies on pure ET and PCET with hydrogen tunnelling pathways to circumvent scaling relations 
often encountered in electrocatalysis.

\section{Acknowledgements}

I acknowledge support by the Alfred Kordelin Foundation and the Academy of Finland (Project No. 307853). I also thank Professor Sharon HammesSchiffer, Dr. Alexander Soudackov, Dr. Yan Choi Lam, and Mr. Zachary Goldsmith for hosting my visit to the Hammes-Schiffer group at Yale, for the useful discussions and help on formulating the ET and PCET rates within the grand canonical ensemble. Computational resources were provided by CSC IT CENTER FOR SCIENCE LTD.

\section{Declaration of interest}

Declarations of interest: none

\section{References}

[1] Z. W. Seh, J. Kibsgaard, C. F. Dickens, I. Chorkendorff, J. K. Nørskov, T. F. Jaramillo, Combining theory and experiment in electrocatalysis: Insights into materials design, Science 355 (2017).

[2] L. R. F. Allen J. Bard, Electrochemical Methods: Fundamentals and Applications, 2nd Edition, John Wiley \& Sons, 2001.

[3] M. M. Melander, M. J. Kuisma, T. E. K. Christensen, K. Honkala, Grand-canonical approach to density functional theory of electrocatalytic systems: Thermodynamics of solid-liquid interfaces at constant ion and electrode potentials, The Journal of Chemical Physics 150 (2019) 041706.

[4] N. D. Mermin, Thermal properties of the inhomogeneous electron gas, Phys. Rev. 137 (1965) A1441-A1443.

[5] A. Pribram-Jones, S. Pittalis, E. K. U. Gross, K. Burke, Thermal density functional theory in context, in: F. Graziani, M. P. Desjarlais, R. Redmer, S. B. Trickey (Eds.), Frontiers and Challenges in Warm Dense Matter, Springer International Publishing, 2014, pp. 25-60. 
[6] R. Evans, The nature of the liquid-vapour interface and other topics in the statistical mechanics of non-uniform, classical fluids, Advances in Physics 28 (1979) 143-200.

[7] T. Kreibich, R. van Leeuwen, E. K. U. Gross, Multicomponent densityfunctional theory for electrons and nuclei, Phys. Rev. A 78 (2008) 022501 .

[8] J. F. Capitani, R. F. Nalewajski, R. G. Parr, Non-oppenheimer density functional theory of molecular systems, The Journal of Chemical Physics 76 (1982) 568-573.

[9] J.-P. H. J.-P. Hansen, Theory of Simple Liquids, 3rd Edition, Academic Press, 2006.

[10] R. Sundararaman, W. A. GoddardIII, T. A. Arias, Grand canonical electronic density-functional theory: Algorithms and applications to electrochemistry, The Journal of Chemical Physics 146 (2017) 114104.

[11] C. D. Taylor, S. A. Wasileski, J.-S. Filhol, M. Neurock, First principles reaction modeling of the electrochemical interface: Consideration and calculation of a tunable surface potential from atomic and electronic structure, Phys. Rev. B 73 (2006) 165402.

[12] J. D. Goodpaster, A. T. Bell, M. Head-Gordon, Identification of possible pathways for $\mathrm{c}-\mathrm{c}$ bond formation during electrochemical reduction of co2: New theoretical insights from an improved electrochemical model, The Journal of Physical Chemistry Letters 7 (2016) 1471-1477. PMID: 27045040.

[13] M. Otani, O. Sugino, First-principles calculations of charged surfaces and interfaces: A plane-wave nonrepeated slab approach, Phys. Rev. B 73 (2006) 115407.

[14] R. Jinnouchi, A. B. Anderson, Electronic structure calculations of liquid-solid interfaces: Combination of density functional theory and modified poisson-boltzmann theory, Phys. Rev. B 77 (2008) 245417.

[15] E. Skulason, V. Tripkovic, M. E. Bjørketun, S. Gudmundsdóttir, G. Karlberg, J. Rossmeisl, T. Bligaard, H. Jónsson, J. K. Nørskov, 
Modeling the electrochemical hydrogen oxidation and evolution reactions on the basis of density functional theory calculations, The Journal of Physical Chemistry C 114 (2010) 18182-18197.

[16] K. Letchworth-Weaver, T. A. Arias, Joint density functional theory of the electrode-electrolyte interface: Application to fixed electrode potentials, interfacial capacitances, and potentials of zero charge, Phys. Rev. B 86 (2012) 075140.

[17] Y.-H. Fang, Z.-P. Liu, Mechanism and tafel lines of electro-oxidation of water to oxygen on ruo2(110), Journal of the American Chemical Society 132 (2010) 18214-18222. PMID: 21133410.

[18] E. Skulason, G. S. Karlberg, J. Rossmeisl, T. Bligaard, J. Greeley, H. Jónsson, J. K. Nørskov, Density functional theory calculations for the hydrogen evolution reaction in an electrochemical double layer on the pt(111) electrode, Phys. Chem. Chem. Phys. 9 (2007) 3241-3250.

[19] K. Chan, J. K. Nørskov, Electrochemical barriers made simple, The Journal of Physical Chemistry Letters 6 (2015) 2663-2668. PMID: 26266844.

[20] G. Kastlunger, P. Lindgren, A. A. Peterson, Controlled-potential simulation of elementary electrochemical reactions: Proton discharge on metal surfaces, The Journal of Physical Chemistry C 122 (2018) 1277112781.

[21] A. Ignaczak, R. Nazmutdinov, A. Goduljan, L. M. de Campos Pinto, F. Juarez, P. Quaino, G. Belletti, E. Santos, W. Schmickler, Oxygen reduction in alkaline media - a discussion, Electrocatalysis 8 (2017) $554-564$.

[22] Z. K. Goldsmith, Y. C. Lam, A. V. Soudackov, S. Hammes-Schiffer, Proton discharge on a gold electrode from triethylammonium in acetonitrile: Theoretical modeling of potential-dependent kinetic isotope effects, Journal of the American Chemical Society 141 (2019) 10841090 .

[23] K. Sakaushi, A. Lyalin, T. Taketsugu, K. Uosaki, Quantum-to-classical transition of proton transfer in potential-induced dioxygen reduction, Phys. Rev. Lett. 121 (2018) 236001. 
[24] A. Ignaczak, R. Nazmutdinov, A. Goduljan, L. M. de Campos Pinto, F. Juarez, P. Quaino, E. Santos, W. Schmickler, A scenario for oxygen reduction in alkaline media, Nano Energy 29 (2016) 362 - 368. Electrocatalysis.

[25] D. Malko, A. Kucernak, Kinetic isotope effect in the oxygen reduction reaction (orr) over fe-n/c catalysts under acidic and alkaline conditions, Electrochemistry Communications 83 (2017) 67 - 71.

[26] E. C. M. Tse, J. A. Varnell, T. T. H. Hoang, A. A. Gewirth, Elucidating proton involvement in the rate-determining step for $\mathrm{pt} / \mathrm{pd}$-based and non-precious-metal oxygen reduction reaction catalysts using the kinetic isotope effect, The Journal of Physical Chemistry Letters 7 (2016) 3542-3547. PMID: 27550191.

[27] V. J. Bukas, H. W. Kim, R. Sengpiel, K. Knudsen, J. Voss, B. D. McCloskey, A. C. Luntz, Combining experiment and theory to unravel the mechanism of two-electron oxygen reduction at a selective and active co-catalyst, ACS Catalysis 8 (2018) 11940-11951.

[28] H. W. Kim, M. B. Ross, N. Kornienko, L. Zhang, J. Guo, P. Yang, B. D. McCloskey, Efficient hydrogen peroxide generation using reduced graphene oxide-based oxygen reduction electrocatalysts, Nature Catalysis 1 (2018) 282-290.

[29] A. J. Gottle, M. T. M. Koper, Proton-coupled electron transfer in the electrocatalysis of $\mathrm{co} 2$ reduction: prediction of sequential vs. concerted pathways using dft, Chem. Sci. 8 (2017) 458-465.

[30] S. Verma, Y. Hamasaki, C. Kim, W. Huang, S. Lu, H.-R. M. Jhong, A. A. Gewirth, T. Fujigaya, N. Nakashima, P. J. A. Kenis, Insights into the low overpotential electroreduction of co2 to co on a supported gold catalyst in an alkaline flow electrolyzer, ACS Energy Letters 3 (2018) 193-198.

[31] M. T. M. Koper, Theory of multiple proton-electron transfer reactions and its implications for electrocatalysis, Chem. Sci. 4 (2013) 2710-2723.

[32] S. Hammes-Schiffer, A. A. Stuchebrukhov, Theory of coupled electron and proton transfer reactions, Chemical Reviews 110 (2010) 6939-6960. PMID: 21049940. 
[33] H. Xiao, T. Cheng, W. A. Goddard, Atomistic mechanisms underlying selectivities in $\mathrm{c} 1$ and $\mathrm{c} 2$ products from electrochemical reduction of co on cu(111), Journal of the American Chemical Society 139 (2017) 130-136. PMID: 28001061.

[34] H. Xiao, T. Cheng, W. A. Goddard, R. Sundararaman, Mechanistic explanation of the ph dependence and onset potentials for hydrocarbon products from electrochemical reduction of co on cu (111), Journal of the American Chemical Society 138 (2016) 483-486. PMID: 26716884.

[35] H. Zhang, W. A. Goddard, Q. Lu, M.-J. Cheng, The importance of grand-canonical quantum mechanical methods to describe the effect of electrode potential on the stability of intermediates involved in both electrochemical co2 reduction and hydrogen evolution, Phys. Chem. Chem. Phys. 20 (2018) 2549-2557.

[36] Y. Huang, R. J. Nielsen, W. A. Goddard, The reaction mechanism for the hydrogen evolution reaction on the basal plane sulfur vacancy site of mos2 using grand canonical potential kinetics, Journal of the American Chemical Society 0 (0) null.

[37] N. Holmberg, K. Laasonen, Ab initio electrochemistry: Exploring the hydrogen evolution reaction on carbon nanotubes, The Journal of Physical Chemistry C 119 (2015) 16166-16178.

[38] S. A. Akhade, N. J. Bernstein, M. R. Esopi, M. J. Regula, M. J. Janik, A simple method to approximate electrode potential-dependent activation energies using density functional theory, Catalysis Today 288 (2017) 63 - 73. Electrochemical Reduction of Carbon Dioxide by heterogenous and homogeneous catalysts: Experiment and Theory.

[39] V. Tripkovic, M. E. Björketun, E. Skúlason, J. Rossmeisl, Standard hydrogen electrode and potential of zero charge in density functional calculations, Phys. Rev. B 84 (2011) 115452.

[40] H.-J. Chun, V. Apaja, A. Clayborne, K. Honkala, J. Greeley, Atomistic insights into nitrogen-cycle electrochemistry: A combined dft and kinetic monte carlo analysis of no electrochemical reduction on pt(100), ACS Catalysis 7 (2017) 3869-3882. 
[41] D. Bohra, I. Ledezma-Yanez, G. Li, W. de Jong, E. A. Pidko, W. A. Smith, Lateral adsorbate interactions inhibit hcoo while promoting co selectivity for co2 electrocatalysis on silver, Angewandte Chemie 131 (2019) 1359-1363.

[42] A. Ignaczak, R. Nazmutdinov, A. Goduljan, L. M. de Campos Pinto, F. Juarez, P. Quaino, G. Belletti, E. Santos, W. Schmickler, Oxygen reduction in alkaline media - a discussion, Electrocatalysis 8 (2017) $554-564$.

[43] A. Goduljan, L. M. de Campos Pinto, F. Juarez, E. Santos, W. Schmickler, Oxygen reduction on $\mathrm{ag}(100)$ in alkaline solutions: A theoretical study, ChemPhysChem 17 (2016) 500-505.

[44] W. Schmickler, E. Santos, M. Bronshtein, R. Nazmutdinov, Adiabatic electron-transfer reactions on semiconducting electrodes, ChemPhysChem 18 (2017) 111-116.

[45] C. Venkataraman, A. V. Soudackov, S. Hammes-Schiffer, Theoretical formulation of nonadiabatic electrochemical proton-coupled electron transfer at metalsolution interfaces, The Journal of Physical Chemistry C 112 (2008) 12386-12397.

[46] I. Navrotskaya, A. V. Soudackov, S. Hammes-Schiffer, Model systembath hamiltonian and nonadiabatic rate constants for proton-coupled electron transfer at electrode-solution interfaces, The Journal of Chemical Physics 128 (2008) 244712.

[47] S. Ghosh, A. V. Soudackov, S. Hammes-Schiffer, Electrochemical electron transfer and proton-coupled electron transfer: Effects of double layer and ionic environment on solvent reorganization energies, Journal of Chemical Theory and Computation 12 (2016) 2917-2925. PMID: 27111050 .

[48] S. Ghosh, S. Horvath, A. V. Soudackov, S. Hammes-Schiffer, Electrochemical solvent reorganization energies in the framework of the polarizable continuum model, Journal of Chemical Theory and Computation 10 (2014) 2091-2102. PMID: 26580536. 
[49] R. Dogonadze, A. Kuznetsov, Theory of charge transfer kinetics at solid-polar liquid interfaces, Progress in Surface Science 6 (1975) 1 41.

[50] R. Dogonadze, 3. theory of molecular electrode kinetics, in: N. Hush (Ed.), Reactions of Molecules at Electrodes, Wiley-Intersciences, 1971, pp. 135-228.

[51] W. Schmickler, A theory of adiabatic electron-transfer reactions, Journal of Electroanalytical Chemistry and Interfacial Electrochemistry 204 (1986) $31-43$.

[52] W. Schmickler, Adiabatic and non-adiabatic electrochemical electron transfer in terms of green's function theory, Russian Journal of Electrochemistry 53 (2017) 1182-1188.

[53] A. Soudackov, S. Hammes-Schiffer, Multistate continuum theory for multiple charge transfer reactions in solution, The Journal of Chemical Physics 111 (1999) 4672-4687.

[54] A. Soudackov, S. Hammes-Schiffer, Derivation of rate expressions for nonadiabatic proton-coupled electron transfer reactions in solution, The Journal of Chemical Physics 113 (2000) 2385-2396.

[55] A. Soudackov, E. Hatcher, S. Hammes-Schiffer, Quantum and dynamical effects of proton donor-acceptor vibrational motion in nonadiabatic proton-coupled electron transfer reactions, The Journal of Chemical Physics 122 (2005) 014505.

[56] R. R. Nazmutdinov, M. D. Bronshtein, E. Santos, Electron transfer across the graphene electrode/solution interface: Interplay between different kinetic regimes, The Journal of Physical Chemistry C 123 (2019) 12346-12354.

[57] D. Borgis, J. T. Hynes, Dynamical theory of proton tunneling transfer rates in solution: general formulation, Chemical Physics 170 (1993) $315-346$.

[58] D. Borgis, J. T. Hynes, Curve crossing formulation for proton transfer reactions in solution, The Journal of Physical Chemistry 100 (1996) $1118-1128$. 
[59] R. I. Cukier, Proton-coupled electron transfer reactions: evaluation of rate constants, The Journal of Physical Chemistry 100 (1996) 1542815443.

[60] N. Ananth, T. F. M. III, Flux-correlation approach to characterizing reaction pathways in quantum systems: a study of condensed-phase proton-coupled electron transfer, Molecular Physics 110 (2012) 10091015.

[61] J. S. Kretchmer, T. F. Miller, Direct simulation of proton-coupled electron transfer across multiple regimes, The Journal of Chemical Physics 138 (2013) 134109.

[62] W. H. Miller, Quantum mechanical transition state theory and a new semiclassical model for reaction rate constants, The Journal of Chemical Physics 61 (1974) 1823-1834.

[63] W. H. Miller, S. D. Schwartz, J. W. Tromp, Quantum mechanical rate constants for bimolecular reactions, The Journal of Chemical Physics 79 (1983) 4889-4898.

[64] W. H. Miller, Direct and correct calculation of canonical and microcanonical rate constants for chemical reactions, The Journal of Physical Chemistry A 102 (1998) 793-806.

[65] R. A. Marcus, On the theory of electron transfer reactions. vi. unified treatment for homogeneous and electrode reactions, The Journal of Chemical Physics 43 (1965) 679-701.

[66] R. v. L. Gianluca Stefanucci, Nonequilibrium Many-Body Theory pf Quantum Systems: A Modern Introduction, Cambridge University Press, pp. 81-124.

[67] A. Agarwal, J. Zhu, C. Hartmann, H. Wang, L. D. Site, Molecular dynamics in a grand ensemble: Bergmannlebowitz model and adaptive resolution simulation, New Journal of Physics 17 (2015) 083042.

[68] M. Tuckerman, Statistical Mechanics: Theory and Molecular Simulations, Oxford University Press, 2010. 
[69] M. H. Peters, An extended liouville equation for variable particle number systems, eprint arXiv:physics/9809039 (1998).

[70] W. H. Miller, Semiclassical limit of quantum mechanical transition state theory for nonseparable systems, The Journal of Chemical Physics 62 (1975) 1899-1906.

[71] A. Agarwal, L. Delle Site, Path integral molecular dynamics within the grand canonical-like adaptive resolution technique: Simulation of liquid water, The Journal of Chemical Physics 143 (2015) 094102.

[72] S. Habershon, D. E. Manolopoulos, T. E. Markland, T. F. Miller, Ringpolymer molecular dynamics: Quantum effects in chemical dynamics from classical trajectories in an extended phase space, Annual Review of Physical Chemistry 64 (2013) 387-413. PMID: 23298242.

[73] J. O. Richardson, M. Thoss, Non-oscillatory flux correlation functions for efficient nonadiabatic rate theory, The Journal of Chemical Physics 141 (2014) 074106.

[74] J. O. Richardson, M. Thoss, Communication: Nonadiabatic ringpolymer molecular dynamics, The Journal of Chemical Physics 139 (2013) 031102.

[75] T. J. H. Hele, S. C. Althorpe, An alternative derivation of ring-polymer molecular dynamics transition-state theory, The Journal of Chemical Physics 144 (2016) 174107.

[76] S. C. Althorpe, T. J. H. Hele, Derivation of a true $(t \rightarrow 0+)$ quantum transition-state theory. ii. recovery of the exact quantum rate in the absence of recrossing, The Journal of Chemical Physics 139 (2013) 084115.

[77] J. O. Richardson, S. C. Althorpe, Ring-polymer molecular dynamics rate-theory in the deep-tunneling regime: Connection with semiclassical instanton theory, The Journal of Chemical Physics 131 (2009) 214106.

[78] J. O. Richardson, Ring-polymer instanton theory, International Reviews in Physical Chemistry 37 (2018) 171-216. 
[79] N. E. Henriksen, F. Y. Hansen, Transition-state theory and dynamical corrections, Phys. Chem. Chem. Phys. 4 (2002) 5995-6000.

[80] J. O. Richardson, R. Bauer, M. Thoss, Semiclassical green's functions and an instanton formulation of electron-transfer rates in the nonadiabatic limit, The Journal of Chemical Physics 143 (2015) 134115.

[81] D. Chandler, Statistical mechanics of isomerization dynamics in liquids and the transition state approximation, The Journal of Chemical Physics 68 (1978) 2959-2970.

[82] G. Henkelman, B. P. Uberuaga, H. Jónsson, A climbing image nudged elastic band method for finding saddle points and minimum energy paths, The Journal of Chemical Physics 113 (2000) 9901-9904.

[83] A. Warshel, R. M. Weiss, An empirical valence bond approach for comparing reactions in solutions and in enzymes, Journal of the American Chemical Society 102 (1980) 6218-6226.

[84] S. C. L. Kamerlin, A. Warshel, The empirical valence bond model: theory and applications, Wiley Interdisciplinary Reviews: Computational Molecular Science 1 (2011) 30-45.

[85] U. W. Schmitt, G. A. Voth, Multistate empirical valence bond model for proton transport in water, The Journal of Physical Chemistry B 102 (1998) 5547-5551.

[86] R. Vuilleumier, D. Borgis, Quantum dynamics of an excess proton in water using an extended empirical valence-bond hamiltonian, The Journal of Physical Chemistry B 102 (1998) 4261-4264.

[87] Q. Wu, T. Van Voorhis, Direct optimization method to study constrained systems within density-functional theory, Phys. Rev. A 72 (2005) 024502.

[88] Q. Wu, T. Van Voorhis, Extracting electron transfer coupling elements from constrained density functional theory, J. Chem. Phys. 125 (2006) 164105.

[89] B. Kaduk, T. Kowalczyk, T. V. Voorhis, Constrained density functional theory, Chem. Rev. 112 (2012) 321-370. 
[90] L. Zusman, Outer-sphere electron transfer in polar solvents, Chemical Physics 49 (1980) 295 - 304.

[91] A. Warshel, Dynamics of reactions in polar solvents. semiclassical trajectory studies of electron-transfer and proton-transfer reactions, The Journal of Physical Chemistry 86 (1982) 2218-2224.

[92] J. Blumberger, Recent advances in the theory and molecular simulation of biological electron transfer reactions, Chemical Reviews 115 (2015) 11191-11238. PMID: 26485093.

[93] A. Nitzan, Chemical Dynamics in Condendsed Phases: Relaxation, Transfer, and Reactions in Condensed Molecular Systems, Oxford University Press, 2006.

[94] R. R. Dogonadze, A. M. Kuznetsov, A. A. Chernenko, Theory of homogeneous and heterogeneous electronic processes in liquids, Russ. Chem. Rev. 34 (1965) 759.

[95] S. K. J.O.M Bockris, Quantum Electrochemistry, Plenum Press, 1979.

[96] R. W. Zwanzig, High-temperature equation of state by a perturbation method. i. nonpolar gases, The Journal of Chemical Physics 22 (1954) 1420-1426.

[97] M. Tachiya, Relation between the electron-transfer rate and the free energy change of reaction, The Journal of Physical Chemistry 93 (1989) 7050-7052.

[98] Y. Tateyama, J. Blumberger, M. Sprik, I. Tavernelli, Densityfunctional molecular-dynamics study of the redox reactions of two anionic, aqueous transition-metal complexes, The Journal of Chemical Physics 122 (2005) 234505.

[99] R. Vuilleumier, K. A. Tay, G. Jeanmairet, D. Borgis, A. Boutin, Extension of marcus picture for electron transfer reactions with large solvation changes, Journal of the American Chemical Society 134 (2012) 2067-2074. PMID: 22148250.

[100] D. A. Rose, I. Benjamin, Molecular dynamics of adiabatic and nonadiabatic electron transfer at the metalwater interface, The Journal of Chemical Physics 100 (1994) 3545-3555. 
[101] H.-. Zhou, A. Szabo, Microscopic formulation of marcus theory of electron transfer, The Journal of Chemical Physics 103 (1995) 34813494.

[102] G. King, A. Warshel, Investigation of the free energy functions for electron transfer reactions, The Journal of Chemical Physics 93 (1990) 8682-8692.

[103] J. Mattiat, J. O. Richardson, Effects of tunnelling and asymmetry for system-bath models of electron transfer, The Journal of Chemical Physics 148 (2018) 102311.

[104] A. Warshel, J. K. Hwang, J. Åqvist, Computer simulations of enzymatic reactions: examination of linear free-energy relationships and quantum-mechanical corrections in the initial proton-transfer step of carbonic anhydrase, Faraday Discuss. 93 (1992) 225-238.

[105] E. Rosta, A. Warshel, Origin of linear free energy relationships: Exploring the nature of the off-diagonal coupling elements in sn2 reactions, Journal of Chemical Theory and Computation 8 (2012) 3574-3585. PMID: 23329895.

[106] S. Trasatti, The absolute electrode potential the end of the story, Electrochimica Acta 35 (1990) 269 - 271.

[107] S. Trasatti, The absolute electrode potential: an explanatory note (recommendations 1986), Journal of Electroanalytical Chemistry and Interfacial Electrochemistry 209 (1986) 417 - 428.

[108] S. Fletcher, The theory of electron transfer, Journal of Solid State Electrochemistry 14 (2010) 705-739.

[109] S. Fletcher, Tafel slopes from first principles, Journal of Solid State Electrochemistry 13 (2009) 537-549.

[110] R. Parsons, General equations for the kinetics of electrode processes, Trans. Faraday Soc. 47 (1951) 1332-1344.

[111] J. K. Nørskov, J. Rossmeisl, A. Logadottir, L. Lindqvist, J. R. Kitchin, T. Bligaard, H. Jónsson, Origin of the overpotential for oxygen reduction at a fuel-cell cathode, The Journal of Physical Chemistry B 108 (2004) 17886-17892. 
[112] N. G. Hörmann, O. Andreussi, N. Marzari, Grand canonical simulations of electrochemical interfaces in implicit solvation models, The Journal of Chemical Physics 150 (2019) 041730.

[113] C. E. D. CHIDSEY, Free energy and temperature dependence of electron transfer at the metal-electrolyte interface, Science 251 (1991) 919-922.

[114] R. Kubo, Generalized Cumulant Expansion Method, Journal of the Physical Society of Japan 17 (1962) 1100-1120.

[115] V. May, O. Kühn, Charge and Energy Transfer Dynamics in Molecular Systems, volume 3rd, WILEY-VCH Verlag GmbH \& Co. KGaA, Weinheim, 2011.

[116] S. Hammes-Schiffer, Proton-coupled electron transfer: classification scheme and guide to theoretical methods, Energy Environ. Sci. 5 (2012) 7696-7703.

[117] Y. Georgievskii, A. A. Stuchebrukhov, Concerted electron and proton transfer: Transition from nonadiabatic to adiabatic proton tunneling, The Journal of Chemical Physics 113 (2000) 10438-10450.

[118] J. H. Skone, A. V. Soudackov, S. Hammes-Schiffer, Calculation of vibronic couplings for phenoxyl/phenol and benzyl/toluene self-exchange reactions: implications for proton-coupled electron transfer mechanisms, Journal of the American Chemical Society 128 (2006) 1665516663. PMID: 17177415.

[119] Y.-C. Lam, A. V. Soudackov, Z. K. Goldsmith, S. Hammes-Schiffer, Theory of proton discharge on metal electrodes: Electronically adiabatic model, The Journal of Physical Chemistry C 123 (2019) 1233512345 .

[120] H. Park, N. Kumar, M. Melander, T. Vegge, J. M. Garcia Lastra, D. J. Siegel, Adiabatic and nonadiabatic charge transport in li-s batteries, Chemistry of Materials 30 (2018) 915-928.

[121] N. Bonnet, T. Morishita, O. Sugino, M. Otani, First-principles molecular dynamics at a constant electrode potential, Phys. Rev. Lett. 109 (2012) 266101. 
[122] A. M. Souza, I. Rungger, C. D. Pemmaraju, U. Schwingenschloegl, S. Sanvito, Constrained-dft method for accurate energy-level alignment of metal/molecule interfaces, Phys. Rev. B 88 (2013) 165112.

[123] Q. Wu, B. Kaduk, T. Van Voorhis, Constrained density functional theory based configuration interaction improves the prediction of reaction barrier heights, J. Chem. Phys. 130 (2009) 034109.

[124] J. Řezáǎ, B. Lévy, I. Demachy, A. de la Lande, Robust and efficient constrained dft molecular dynamics approach for biochemical modeling, J. Chem. Theory Comput. 8 (2012) 418-427.

[125] P. Ramos, M. Pavanello, Constrained subsystem density functional theory, Phys. Chem. Chem. Phys. 18 (2016) 21172-21178.

[126] H. Oberhofer, J. Blumberger, Charge constrained density functional molecular dynamics for simulation of condensed phase electron transfer reactions, J. Chem. Phys. 131 (2009) 064101.

[127] H. Oberhofer, J. Blumberger, Electronic coupling matrix elements from charge constrained density functional theory calculations using a plane wave basis set, J. Chem. Phys. 133 (2010) 244105.

[128] P. Ghosh, R. Gebauer, Computational approaches to charge transfer excitations in a zinc tetraphenylporphyrin and c70 complex, J. Chem. Phys. 132 (2010) 104102.

[129] A. M. P. Sena, T. Miyazaki, D. R. Bowler, Linear scaling constrained density functional theory in conquest, J. Chem. Theory Comput. 7 (2011) 884-889.

[130] L. E. Ratcliff, L. Grisanti, L. Genovese, T. Deutsch, T. Neumann, D. Danilov, W. Wenzel, D. Beljonne, J. Cornil, Toward fast and accurate evaluation of charge on-site energies and transfer integrals in supramolecular architectures using linear constrained density functional theory (cdft)-based methods, J. Chem. Theory Comput. 11 (2015) 2077-2086.

[131] M. Melander, E. O. Jónsson, J. J. Mortensen, T. Vegge, J. M. García Lastra, Implementation of constrained dft for computing charge transfer rates within the projector augmented wave method, Journal 
of Chemical Theory and Computation 12 (2016) 5367-5378. PMID: 27749068.

[132] N. Holmberg, K. Laasonen, Efficient constrained density functional theory implementation for simulation of condensed phase electron transfer reactions, Journal of Chemical Theory and Computation 13 (2017) 587-601. PMID: 28009515.

[133] D. J. Tannor, Introduction to Quantum Mechanics: A Time-Dependent Perspective, University Science Books, 2007.

[134] E. Santos, A. Lundin, K. Pötting, P. Quaino, W. Schmickler, Model for the electrocatalysis of hydrogen evolution, Phys. Rev. B 79 (2009) 235436 .

[135] E. Santos, M. Koper, W. Schmickler, Bond-breaking electron transfer of diatomic reactants at metal electrodes, Chemical Physics 344 (2008) $195-201$.

[136] A. Ignaczak, W. Schmickler, Theoretical study of a non-adiabatic dissociative electron transfer reaction, Journal of Electroanalytical Chemistry 554-555 (2003) 201 - 209. Special issue in memory of Professor M.J. Weaver.

[137] N. Holmberg, K. Laasonen, Diabatic model for electrochemical hydrogen evolution based on constrained dft configuration interaction, The Journal of Chemical Physics 149 (2018) 104702.

[138] Q. Wu, B. Kaduk, T. Van Voorhis, Constrained density functional theory based configuration interaction improves the prediction of reaction barrier heights, The Journal of Chemical Physics 130 (2009) 034109.

[139] Q. Wu, C.-L. Cheng, T. Van Voorhis, Configuration interaction based on constrained density functional theory: A multireference method, The Journal of Chemical Physics 127 (2007) 164119.

[140] I. Rips, J. Jortner, Dynamic solvent effects on outersphere electron transfer, The Journal of Chemical Physics 87 (1987) 2090-2104.

[141] R. F. Grote, J. T. Hynes, The stable states picture of chemical reactions. ii. rate constants for condensed and gas phase reaction models, The Journal of Chemical Physics 73 (1980) 2715-2732. 
[142] A. K. Mishra, D. H. Waldeck, A unified model for the electrochemical rate constant that incorporates solvent dynamics, The Journal of Physical Chemistry C 113 (2009) 17904-17914.

[143] J. T. Hynes, Outer-sphere electron-transfer reactions and frequencydependent friction, The Journal of Physical Chemistry 90 (1986) 37013706 .

[144] V. Gladkikh, A. I. Burshtein, I. Rips, Variation of the resonant transfer rate when passing from nonadiabatic to adiabatic electron transfer, The Journal of Physical Chemistry A 109 (2005) 4983-4988. PMID: 16833848 .

[145] T. Ikeshoji, M. Otani, Toward full simulation of the electrochemical oxygen reduction reaction on pt using first-principles and kinetic calculations, Phys. Chem. Chem. Phys. 19 (2017) 4447-4453.

[146] M. Van den Bossche, E. Skulason, C. Rose-Petruck, H. Jónsson, Assessment of constant-potential implicit solvation calculations of electrochemical energy barriers for h2 evolution on pt, The Journal of Physical Chemistry C 0 (0) null.

[147] Z.-F. Huang, J. Wang, Y. Peng, C.-Y. Jung, A. Fisher, X. Wang, Design of efficient bifunctional oxygen reduction/evolution electrocatalyst: Recent advances and perspectives, Advanced Energy Materials 7 (2017) 1700544-n/a. 1700544.

[148] S. Back, Y. Jung, Importance of ligand effects breaking the scaling relation for coreshell oxygen reduction catalysts, ChemCatChem 9 (2017) 3173-3179.

[149] A. Kulkarni, S. Siahrostami, A. Patel, J. K. Nørskov, Understanding catalytic activity trends in the oxygen reduction reaction, Chemical Reviews 0 (0) null. PMID: 29405702. 